\title{
Control-volume distributed multi-point flux approximation coupled with a lower-dimensional fracture model
}

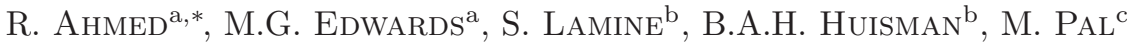 \\ ${ }^{a} Z C C E$, College of Engineering, Swansea University, Swansea SA2 8PP, Wales UK \\ ${ }^{b}$ Shell Global Solutions International B.V., Kessler Park 1, 2288 GS, Rijswijk, The Netherlands. \\ ${ }^{c}$ Maersk Oil and Gas A/S, 1263 Copenhagen, Denmark.
}

\begin{abstract}
A cell-centered control-volume distributed multi-point flux approximation (CVD-MPFA) finite-volume formulation is presented for discrete fracture-matrix simulations. The grid is aligned with the fractures and barriers which are then modeled as lower-dimensional interfaces located between the matrix cells in the physical domain. The $\mathrm{nD}$ pressure equation is solved in the matrix domain coupled with an (n-1)D pressure equation solved in the fractures. The CVD-MPFA formulation naturally handles fractures with anisotropic permeabilities on unstructured grids. Matrix-fracture fluxes are expressed in terms of matrix and fracture pressures, and must be added to the lower-dimensional flow equation (called the transfer function). An additional transmission condition is used between matrix cells adjacent to low permeable fractures to link the velocity and pressure jump across the fractures. Numerical tests serve to assess the convergence and accuracy of the lower-dimensional fracture model for highly anisotropic fractures having different apertures and permeability tensors. A transport equation for tracer flow is coupled via the Darcy flux for single and intersecting fractures. The lower-dimensional approach for intersecting fractures avoids the more restrictive CFL condition corresponding to the equi-dimensional approximation with explicit time discretization. Lower-dimensional fracture model results are compared with hybrid-grid and equi-dimensional model results. Fractures and barriers are efficiently modeled by lower-dimensional interfaces which yield comparable results to those of the equi-dimensional model. Highly conductive fractures are modeled as lower-dimensional entities without the use of locally refined grids that are required by the equi-dimensional model, while pressure continuity across fractures is built into the model, without depending on the extra degrees of freedom which must be added locally by the hybrid-grid method.The lower-dimensional fracture model also yields improved results when compared to those of the hybrid-grid model for fractures with low-permeability in the normal direction to the fracture. In addition, a transient pressure simulation involving geologically representative complex fracture networks is presented.
\end{abstract}

Keywords: CVD-MPFA, fracture network, lower-dimensional, interfaces, fracture matrix transfer

\footnotetext{
* Corresponding author

Email addresses: R.AHMED.642142@swansea.ac.uk (R. AHMED), m.g.edwards@swansea.ac.uk (M.G. EDWARDS)
} 


\section{Introduction}

Understanding of fluid flow through a fractured porous medium has immense importance in energy production and environmental problems. The oil industry has a special interest because an estimated $60 \%$ of the world's remaining oil reserves reside in fractured formations [1]. In addition to oil and gas production

5 ment in the subsurface [2], and flow of non-aqueous-phase liquids in aquifers. Fractures are a system of rock discontinuities e.g. faults, joints and fissures, that occur in porous media with apertures having widths ranging over scales from microns to centimeters [3]. Open fractures act as preferential fluid flow paths above a certain aperture and size whereas cemented fractures can act as flow barriers. Flow, in any rock, is affected length scales ranging from microns to hundreds of kilometers [4, 5]. Usually the matrix provides the storage for the fluid while fractures provide the main fluid flow paths. For example in two-phase flow, fractures may form the predominant flow paths for a particular phase and the less permeable matrix may become the flow region for the other phase [6].

15

The importance of fractures in reservoirs has lead to increasing effort being devoted to development of efficient and accurate numerical methods to simulate fluid flow through fractured porous media. Dualporosity/permeability models, developed in [7, 8, 9], have traditionally been used for the last few decades. Flow transfer terms are defined between the fracture and matrix systems. These transfer terms depend on the shape factor, average pressure difference between two domains and further physical parameters in the case of multi-phase flow [10]. The shape factor is not straightforward to determine and is not available in the presence of capillarity and gravity for two-phase flow[2]. Also, barriers cannot be modeled by dualporosity/permeability models. Moreover, these models are based on the assumption that fracture systems are dense so inaccurate results are given for large scale fractures. As a result, the discrete-fracture model (DFM) was developed; see e.g. 11, 12, 13, 14, 15, 16], which is attractive for large scale and sparse fracture systems. In this model actual geometry and location of the fracture are honored in the domain. Unlike the dual-porosity model; the effect of individual fractures on fluid flow can be determined and fluid transfer between the fracture and matrix is more straightforward and consistent. Generally, fractures are modeled by ( $\mathrm{n}-1)$ dimensional elements in a $\mathrm{n}$-dimensional domain, for example in $2 \mathrm{D}$, fractures are represented by lines at the edges of the polygonal matrix elements. Equi-dimensional representation of fractures [17], are not popular because of complexity and computational cost contributed by thin cells. In the equi-dimensional model, the control-volume at the intersection of the fractures is of the dimensions of fracture aperture which reduces the time-step size in the numerical model [2]. Also, in our experience with this method we have observed that a small control-volume increases the condition number of the global linear system which increases the computational cost for the solution of the system, consistent with [18].

In the discrete-fracture method, rock-matrix and fracture elements coincide at the interface, so an un- 
structured grid is used to honor the explicit fracture geometry(see 19, 20]). Also, the matrix cells near the fracture are small enough to conform to the fracture geometry. Small cells lead to a large numerical system to be solved. Because of grid conformity, this model may not be applied to dynamic fracture network modeling where the grid is updated because of generation of new fractures [21, p. 72]. Moreover, the discrete-fracture model may not be used for small scale fractures and any cases with large numbers of fractures, which would lead to locally dense unstructured grids in-turn leading to high computational cost. A hybrid method(fracture-only model) that gets advantages of both dual-porosity method and discrete-fracture method has been presented in [22]. Hierarchical fracture models have been presented in [23, 24] for flow simulation in a fractured porous medium. In this approach, small scale fractures are homogenized into the matrix medium and their effects are added to the matrix permeability. Large scale fractures are explicitly modeled as major fluid conduits embedded in non-conforming structured meshes. Other techniques based on discontinuity finite element modeling and extended-finite element method(XFEM) for embedded fractures into non-conforming mesh are presented in [25, 26, 27]. Recently, a continuum voxel approach has been presented in 28] where hydraulic properties of a fracture network are mapped onto a stair-like regular mesh to avoid the intense meshing issue for discrete-fractures. A technique of multi-scale philosophy is presented in [29] to reduce the number of degrees of freedom for fracture-only simulations. Various numerical methods have been used with DFM for single and multiphase flow in fractured porous media. Mass conservative methods include control-volume finite-element (CVFE), see [6], cell-centered finite-volume (CCFV) [14, 18] and mixed finite-element (MFE) [16, 2, 26]. CVFE is not control volume distributed and is not flux-continuous for heterogeneous porous medium. MFE is locally flux-continuous and consistent but is computationally expensive because of higher degrees of freedom per cell as compared to CCFV and CVFE.

Herein, we will focus on a locally conservative cell-centered finite-volume (CCFV) formulation coupled with discrete-fracture networks, in particular we use the control-volume distributed multi-point flux approximation (CVD-MPFA) [30]. We choose CVD-MPFA because it is flux-continuous and consistent for heterogeneous porous media and uses a single degree of freedom per control-volume (grid cell in this case). Note that commercial simulators also use a single degree of freedom per grid cell. We use a conforming unstructured mesh to capture the heterogeneity of a porous medium with fractures. Recently, Sandve et al [18] presented CVDMPFA, so-called O-method (TPS $q=1$ ), for discrete-fracture and matrix simulation based on hybrid-grid approach [14]. In the hybrid-grid approach, fractures are (n-1)D in the physical mesh and are expanded to $\mathrm{nD}$ in the computational domain. The $\mathrm{nD}$ pressure equation is solved by the usual CVD-MPFA formulation in both matrix and fractures in the computational domain. The main difference between equi-dimensional model and hybrid-grid model is the treatment of intermediate cell between the intersecting fractures. In a hybrid-grid, the intermediate cell is assumed to be of small size so that pressure variation is zero in that cell to avoid the complexity that would be incurred by the small size of intermediate cell in the equi-dimensional model.

In this work, we present and investigate the possibility of an alternative and simplified CVD-MPFA formu- 
lation where fractures are modeled as lower-dimensional cells in the computational domain as well, unlike the hybrid-grid method where special 2D hybrid fracture cells are required. In the formulation presented here, an $\mathrm{nD}$ pressure equation is solved in the matrix domain coupled with a fracture network where an (n-1)D pressure equation is solved. We refer to this method as a lower-dimensional fracture model (or 1D fracture model in $2 \mathrm{D}$ context) because fractures are strictly lower-dimensional. The coupled CVD-MPFA formulation can easily be incorporated into current CVD-MPFA based simulators. Moreover, we compare the pressure and transport solutions of the lower-dimensional fracture model and hybrid-grid model with the results of an explicit equi-dimensional model on unstructured meshes. The lower-dimensional fracture model gives comparable results to those given by the hybrid-grid and equi-dimensional models for domains involving fractures and barriers. The assumption of pressure continuity across highly conductive fractures enables the lower-dimensional model to avoid the extra local degrees of freedom required by the hybrid-grid method. The outline of the paper is as follows; we present the flow equations in section 2 and a short description of CVD-MPFA is presented in section 3. We review and discuss the hybrid-grid method in section 4 for fractured media before presenting our CVD-MPFA formulation in section 5 for the 2D matrix coupled with 1D fractures. Numerical tests are presented in section 7 to compare the lower-dimensional fracture, hybridgrid and equi-dimensional models with application to fractured media problems. Conclusions based on the numerical results are given in the last section 8

\section{Flow equations}

We focus on the discretization of an elliptic partial differential equation for pressure by a CVD-MPFA method for DFM. The pressure equation arises from Darcy's law and mass conservation for single phase flow, (a similar method is also applicable to multiphase flow). The resulting elliptic pressure equation

$$
-\nabla \cdot \frac{k}{\mu} \nabla \phi=q_{c}
$$

95 term, $\boldsymbol{k}$ is the permeability tensor and $\mu$ is the viscosity of the fluid. As usual in single phase flow we let $\boldsymbol{K}=\frac{\boldsymbol{k}}{\mu}$ denote the (abbreviated) possibly heterogeneous spatially varying, symmetric permeability tensor of second rank with possibly non-zero off-diagonal coefficients written, in general, as; $\boldsymbol{K}=\left(\begin{array}{cc}K_{11} & K_{12} \\ K_{21} & K_{22}\end{array}\right)$. The Darcy velocity is given by $\boldsymbol{v}=-\boldsymbol{K} \nabla \phi$. Equation (10) is solved here subject to Dirichlet and/or Neumann boundary conditions which are $\phi=h(\boldsymbol{x})$ and $(\boldsymbol{K} \nabla \phi) \cdot \boldsymbol{n}=g(\boldsymbol{x})$ respectively on the domain boundary $\delta \Omega$, where $h$ and $g$ are scalar fields defined at the boundary and $\boldsymbol{n}$ is the normal vector at the boundary.

The permeability tensor in the general axes can be locally transformed to the tangential and normal directions of the fracture and can be written as $\boldsymbol{K}_{\boldsymbol{f}}=\left(\begin{array}{cc}K_{f, t} & 0.0 \\ 0.0 & K_{f, n}\end{array}\right)$. Usually, $K_{f, t} \leq a_{h}^{2} / 12$ which is 
the maximum tangential permeability of the region, of width $a_{h}$, when flow is between two parallel plates

without tortuosity or cementation.

\section{Tracer transport equation}

The mass conservation equation for tracer transport ignoring dispersion is written as the advection equation below:

$$
\varphi \frac{\partial c}{\partial t}+\nabla \cdot(\boldsymbol{v} c)=q_{c}
$$

where, $c$ is the tracer concentration and $\varphi$ is the porosity which can be taken as unity here for simplicity.

\section{CVD-MPFA description}

Here, we review CVD-MPFA method, with triangular pressure support. Details of the CVD-MPFA qfamilies with triangular pressure support can be found in [31, 30, 32], and the default case $q=1$ in [33]. Recently, MPFA method with full pressure support has also been presented in [34, 35, 36]. Following the finite-volume method, the first step is to integrate the flow equation (11) over the control-volume and apply the Gauss divergence theorem to get,

$$
-\oint_{\delta \Omega_{i}}(\boldsymbol{K} \nabla \phi) \cdot \boldsymbol{n} d S=\int_{\Omega_{i}} q_{c} d V
$$

where, $\Omega_{i}$ denotes the boundary of the arbitrary control-volume $\Omega_{i}$ and the $\boldsymbol{n}$ is a normal vector at the boundary $\delta \Omega_{i}$.

For the cell-centered finite-volume method, control-volumes are defined by the grid cells which can be triangles, quadrilaterals or be of any polygonal shape. The numerical solution is associated with the grid point, which is usually the cell centroid, as in this work, but the circumcenter is an obvious alternative for acute triangles. Flow variables and physical properties are assigned to the grid cells, i.e. control-volume distributed (CVD). Continuous flux and pressure constraints are imposed locally with respect to each cluster of cells that are attached to a common grid vertex. A dual-cell is introduced which is defined by connecting each grid point of cell with the mid-points of the edges which are attached to the cluster vertex. Resulting polygon around the cluster vertex is dual-cell. Sub-cell is the quadrilateral formed when dual-cells overlay the primal cell. Each sub-cell is defined by the anticlockwise loop starting from grid point of cell to right-edge midpoint, cluster vertex, left-edge midpoint then back to cell centroid. Edge mid-point divides the cell interface into two segments; referred as sub-interface In CVD-MPFA method, normal flux continuity and pressure continuity are fulfilled for every sub-interface. In this work, we employ the triangle pressure support(TPS) formulation. An auxiliary interface pressure is introduced on each sub-interface to ensure point-wise pressure continuity. For a given dual-cell, position of continuity can be chosen at any point between the edge mid-point and the common grid vertex (but not at the vertex) of the cluster of cells. The continuity point is defined by the parametric variation in $[0<q \leq 1]$ along the sub-interface, which leads to the family of schemes depending 
on the choice of quadrature $q$. The singular point $q=0$ corresponds to the cluster vertex (which is avoided) and $q=1$ corresponds to the edge midpoint and is the standard default method. Here, the symmetric positive definite scheme of [30] with $q=2 / 3$ is used. The cluster and dual-cell are shown in figure 1(a) In

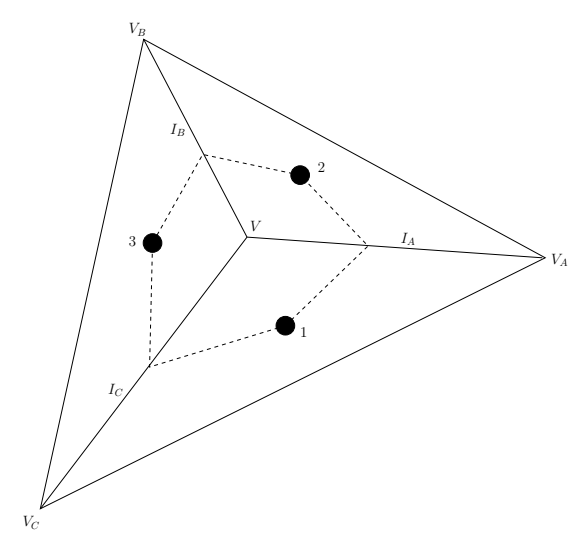

(a) Cluster of triangular cells common to vertex $V$. Dual-cell is depicted by the dashed line polygon.

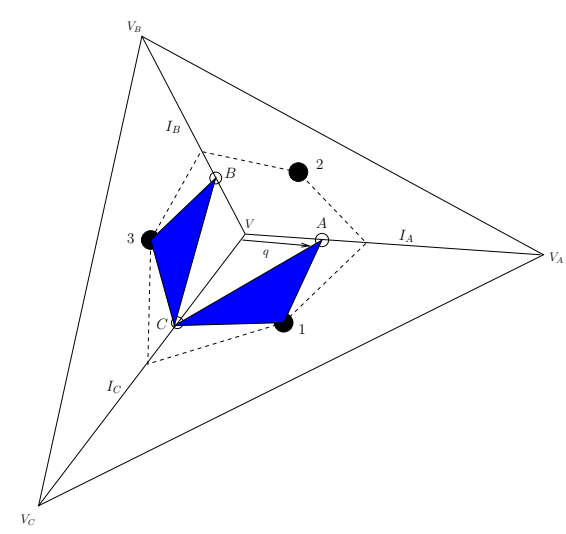

(b) Triangular pressure supports for subcells of cells 1 and 3 .

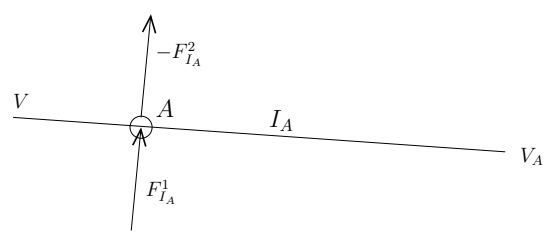

(c) Continuity of flux and pressure on sub-interface $I_{A}$.

Figure 1: Cluster and dual-cell of three triangular cells.

this scheme, pressure is assumed piecewise linear in a triangular region defined by joining cell center with right-edge interface continuity point and left-edge interface continuity point. Pressure in the triangular region of cell 1 is written in terms of barycentric coordinate referential $(\xi, \eta)$ as $\phi=(1-\xi-\eta) \phi_{1}+\xi \phi_{A}+\eta \phi_{C}$. A piecewise constant pressure gradient vector can be formed over each sub-cell from the triangular linear pressure field from which the Darcy velocity vector can be determined in each sub-cell. The Darcy velocity is resolved along the two outward sub-interfaces of the sub-cell. So, the normal flux at the left hand side of sub-interface $I_{A}$ is given by velocity resolution along the normal vector $d L_{h}=0.5\left(\left(y_{V}-y_{V_{A}}\right),-\left(x_{V}-x_{V_{A}}\right)\right)$ outward to cell 1 as,

$$
F_{I_{A}}^{1}=\boldsymbol{v}_{h} \cdot d L_{h}=-\left.\left(T_{11}^{1} \phi_{\xi}+T_{12}^{1} \phi_{\eta}\right)\right|_{A} ^{1}
$$

where, $\boldsymbol{T}=\boldsymbol{T}(q)$ is known as the general Piola tensor and define the coefficients of $\left(\phi_{\xi}, \phi_{\eta}\right)^{T}$. For the full definition of general tensor we refer to [30]. Similarly, fluxes are determined on other sub-interfaces as well. Flux continuity is imposed on every sub-interface e.g, for $I_{A}$ flux continuity is written as,

$$
\begin{aligned}
F_{I_{A}}^{1} & =-F_{I_{A}}^{2} \\
-\left.\left(T_{11}^{1} \phi_{\xi}+T_{12}^{1} \phi_{\eta}\right)\right|_{I_{A}} ^{1} & =\left.\left(T_{21}^{2} \phi_{\xi}+T_{22}^{2} \phi_{\eta}\right)\right|_{I_{A}} ^{2}
\end{aligned}
$$


All the flux continuity conditions, combined, can be written in matrix form as follows

$$
\boldsymbol{F}=\boldsymbol{A}_{L}^{3 \times 3} \Phi_{m}+\boldsymbol{B}_{L}^{3 \times 3} \Phi_{I}=\boldsymbol{A}_{R}^{3 \times 3} \Phi_{m}+\boldsymbol{B}_{R}^{3 \times 3} \Phi_{I}
$$

where $\Phi_{m}=\left(\phi_{1}, \phi_{2}, \phi_{3}\right)^{T}$ and $\Phi_{I}=\left(\phi_{A}, \phi_{B}, \phi_{C}\right)^{T}$. Using condition (6), we can eliminate $\Phi_{I}$ to get the

fluxes in terms of $\Phi_{m}$ given as,

$$
\boldsymbol{F}=\left(\boldsymbol{A}_{L}+\boldsymbol{B}_{L}\left(\boldsymbol{B}_{R}-\boldsymbol{B}_{L}\right)^{-1}\left(\boldsymbol{A}_{L}-\boldsymbol{A}_{R}\right)\right) \Phi_{m}
$$

In this way, we determine fluxes on all the sub-interfaces related to a given cell in terms of grid-cell pressures only. The discrete scheme of the equation (3) is then defined by the summation of determined fluxes on all the sub-interfaces related to the given grid cell.

\section{Hybrid CVD-MPFA method for fractured media} model where thin fractures are gridded by small grid cells and are assigned fracture permeability for computations. The CVD-MPFA is employed to solve the problem on the given mesh as in the case of a heterogeneous medium. Because of the small apertures of fractures, grid generation is complicated and the numerical simulation is costly, specifically in the case of intersecting fractures. The intermediate cells between the intersecting fractures are of dimensions of fracture aperture which increases the condition number of the global linear system and reduces the time-step size limit for the transport problems thus increasing the overall computational cost. To address the problems associated with equi-dimensional fracture modeling, the hybrid-grid method was introduced for cell-centered finite-volume with two-point flux approximation (TPFA) for discrete-fracture model [37]. The TPFA scheme gives inaccurate results when the grid is not Korthogonal. Sandve et. al [18] presented a hybrid-grid method with multi-point flux approximation, so-called O-method, for single-phase flow through fractured porous medium. In the hybrid-grid method, fractures are modeled as lower-dimensional entities in the geometric mesh but are expanded to equi-dimensional cells in computational domain. In 2D, the mesh is conforming with fractures on cell edges, which are expanded to rectangular cells in the computational domain leading to a hybrid-mesh. This is similar to an equidimensional method for a matrix with fractures as discussed in [17], where fractures are expanded from lower-dimensional to equi-dimensional cells after the meshing. The main difference between the hybrid-grid method and equi-dimensional method is in the treatment of adjusted geometry and intermediate cells. In 18], computations are obtained without adjusting the actual area of the neighboring matrix cells when fracture cells are expanded. In the hybrid-grid, unknowns are associated with the centroids of matrix cells and lower-dimensional fracture cells. During computations the fracture is given area which is equal to the length of the edge, between matrix cells, multiplied by the aperture of the fracture. Hybrid cells are represented by the insertion of midpoints of the edges of the fracture cell, which are a half-aperture away on both sides of 
the fracture centroid. There will be a hybrid face where the hybrid fracture cell has a neighboring hybrid cell, as depicted in figure 2(b) by dashed-lines. For intersecting fractures, there is an intermediate cell depicted

fractures and intermediate cell is explicitly constructed then it will lead to full equi-dimensional modeling

A cluster of cells involving fracture cells in a geometric mesh is shown in figure 2(a) Pressure continuity

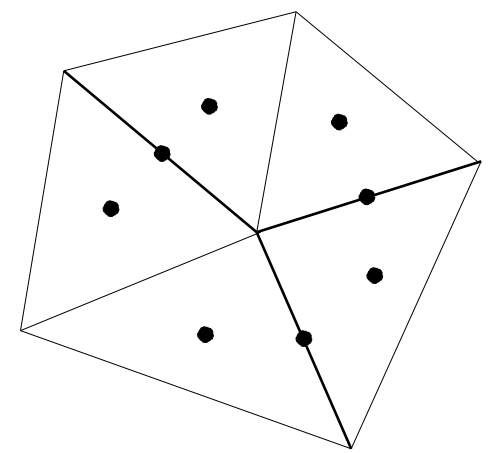

(a) Geometric cluster involving fracture edges, depicted by bold edges. The centroids of the cells are also shown as dots.

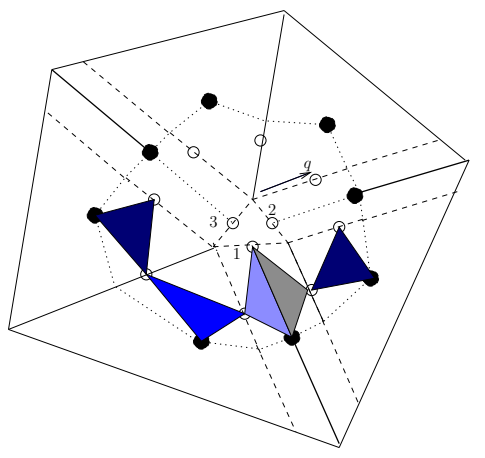

(b) Cluster of hybrid-grid. Triangular pressure supports are also shown in hybrid sub-cells. Pressure points on hybrid faces of intermediate cell are numbered.

Figure 2: Cluster of matrix and fracture cells. Fracture cells are expanded to 2D for computations.

points on the fracture cells adjacent to the matrix sub-cells are moved by half-aperture into the sub-cells in the normal direction to the fractures. In this way fracture cells are expanded on both sides of each fracture and new pressure continuity points are defined between fracture and matrix sub-cells on the so called hybrid faces. The cluster of matrix and fracture sub-cells in the hybrid-grid is shown in figure 2(b), Pressure points on the sub-interfaces and hybrid faces are depicted by circles and primal pressures are depicted by dots. Half-fluxes are computed on all the sub-interfaces for every sub-cell in terms of primal pressure and interface pressures as described in section 3. Continuity of fluxes is imposed on the matrix-matrix and matrix-fracture sub-interface pressure points to eliminate interface pressures on the sub-interfaces. To eliminate pressure on the hybrid faces of an intermediate cell between intersecting fractures, two conditions are imposed. First, pressure on all hybrid faces of an intermediate cell is continuous and second summation of fluxes on these faces is zero. For the dashed-line triangular intermediate cell of cluster shown in figure 2(b), the conditions can be written as:

$\phi_{k}=\phi_{v} \quad k=1,2,3 \quad$ and $\quad \sum_{k=1}^{3} F_{k}=0$

enabling the half-face fluxes to be expressed in terms of local cell pressures, and consequently after assembly 
the finite-volume approximation yields a linear system of equations with solution vector comprised of the global cell-centered pressure field.

\section{CVD-MPFA formulation with coupling for fractures} fracture cells:

where $\nabla_{t}$ and $\boldsymbol{K}_{f, t}$ are the respective longitudinal (tangential) gradient operator and permeability of fracture. $q_{c m}$ and $q_{c f}$ are known source terms for the rock matrix and fracture respectively. Transfer function, $q_{f}$, accounts for the net normal flux transfer between matrix and fracture cells, resulting from the dimensionality reduction to obtain (n-1)D equation(9). Matrix-fracture flux is also added naturally in the formulation for the matrix cells discussed later on. The flow equations (8) and (9) are integrated over the grid cell control 
volumes using the Gauss divergence theorem to obtain,

$$
\begin{aligned}
-\oint_{\delta \Omega_{m i}}\left(\boldsymbol{K}_{m} \nabla \phi\right) \cdot \boldsymbol{n}_{i} d S & =\bar{q}_{c m} \\
-\oint_{\delta \Omega_{f_{j}}}\left(\boldsymbol{K}_{f, t} \nabla_{t} \phi_{f}\right) \cdot \boldsymbol{n}_{j} d S+Q_{f} & =\bar{q}_{c f}
\end{aligned}
$$

where $\bar{q}_{c m}=\int_{\Omega_{m i}} q_{c m} d V$ and $\bar{q}_{c f}=\int_{\Omega_{f}} q_{c f} d V$ are respective specified source terms for the matrix and fracture cells $\Omega_{m i}, \Omega_{f_{j}}$. Moreover, $Q_{f}=\int_{\Omega_{f_{j}}} q_{f} d V$, is the transfer function resulting from the net flux normal to the lower-dimensional fracture cell $\Omega_{f_{j}}$. We introduce the CVD-MPFA formulation for the reduced dimensional fractures in the cluster shown in figure 2(a). The dual cell for the cluster is shown in figure 3 by the dashed-line polygon. The sub-cells are not modified in any way. The half fluxes for the dual cell are also depicted in the figure 3 .

\subsection{Matrix-matrix and matrix-fracture fluxes}

Pressure unknowns are associated with the centroids of the $\mathrm{nD}$ matrix cells. Point-wise pressure continuity is also imposed at all the sub-interfaces between the matrix sub-cells except those interfaces(edges) which are $1 \mathrm{D}$ fracture cells in mesh. Triangular pressure support is defined by the pressure points at cell center, rightedge and left-edge. The pressure points on the edges can be chosen along the sub-interface by quadrature, $[0<q \leq 1]$. For the edges which are fractures, only $q=1$ can be used as these pressures are related to the fracture centroid pressure unknown. Fluxes are computed in an anticlockwise direction on both sides of each of the sub-cells. We describe our CVD-MPFA formulation for the cluster of cells shown in figure 3(a). We have 6 matrix cells and 3 fracture cells as interfaces between the matrix cells. The dual-cell is represented by the dashed-line polygon. Sub-cells and triangular pressure supports are depicted in figure 3(b). Discrete piecewise fluxes on the sub-interfaces can be determined in the same way as shown in equation (4).

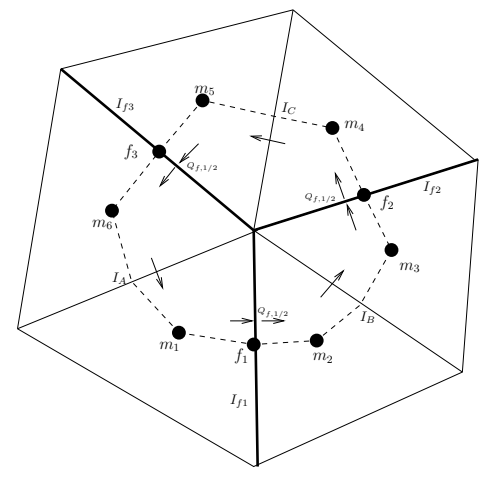

(a) Cluster of matrix cells involving fracture cells as interfaces in between, depicted by bold lines. Fluxes are discontinuous across fracture-interfaces, but pressure is continuous.

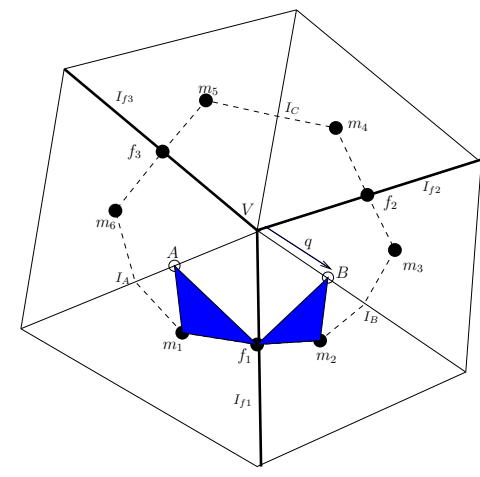

(b) Dual cell with sub-cells of matrix cells. Triangular pressure supports are also shown for matrix cells 1 and 2 .

Figure 3: Cluster and dual-cell involving fracture cells as interfaces 


\subsubsection{Continuous pressure model}

For high permeability and low aperture, the jump in pressure across the fracture is very low. Pressure can be assumed constant across the width of fracture but the velocity jump is not zero. In this case, point-wise pressure continuity is imposed on the sub-interfaces which are $1 \mathrm{D}$ fracture cells. For the sub-cell of cell $m_{1}$ of cluster shown in figure $3(\mathrm{a})$, outward flux on right-edge $I_{f_{1}}$ can be written as,

$$
F_{I_{f 1}}^{1}=-\left.\left(T_{11}^{1} \phi_{\xi}+T_{12}^{1} \phi_{\eta}\right)\right|_{I_{f_{1}}} ^{1}
$$

where,

$$
\left(\begin{array}{c}
\phi_{\xi} \\
\phi_{\eta}
\end{array}\right)=\left(\begin{array}{c}
\phi_{f_{1}}-\phi_{m_{1}} \\
\phi_{A}-\phi_{m_{1}}
\end{array}\right)
$$

Similarly, for sub-cell of cell $m_{2}$, outward flux on left-edge $I_{f_{1}}$ can be written as,

$$
F_{I_{f 1}}^{2}=-\left.\left(T_{21}^{2} \phi_{\xi}+T_{22}^{2} \phi_{\eta}\right)\right|_{I_{f_{1}}} ^{2}
$$

where,

$$
\left(\begin{array}{c}
\phi_{\xi} \\
\phi_{\eta}
\end{array}\right)=\left(\begin{array}{c}
\phi_{B}-\phi_{m_{2}} \\
\phi_{f_{1}}-\phi_{m_{2}}
\end{array}\right)
$$

Fluxes determined in equations (12) and (14) are discontinuous because the sub-interface is half 1D fracture cell $f_{1}$, but the pressure of fracture cell $\phi_{f_{1}}$ is continuous and is unknown. In the same way, fluxes on all other sub-interfaces are determined. For the concerned cluster of cells, we require 3 fluxes on the left side of sub-interfaces which are not fracture and 6 fluxes on both the sides (right and left) of the sub-interfaces which are fractures and can be formulated in the combined simple form of matrices as follows:

$$
\boldsymbol{F}=A^{9 \times 6} \Phi_{m}+B^{9 \times 3} \Phi_{I}+C^{9 \times 3} \Phi_{f}
$$

where $\Phi_{m}=\left(\phi_{m_{1}}, \phi_{m_{2}}, \phi_{m_{3}}, \phi_{m_{4}}, \phi_{m_{5}}, \phi_{m_{6}}\right)^{T}$ are pressures associated with the matrix cells, $\Phi_{I}=\left(\phi_{A}, \phi_{B}, \phi_{C}\right)^{T}$ are pressures on the sub-interfaces, between matrix cells, which are not fracture cells and $\Phi_{f}=\left(\phi_{f_{1}}, \phi_{f_{2}}, \phi_{f_{3}}\right)^{T}$ are pressures associated with the involved fracture cells. As in the usual CVD-MPFA formulation, $\Phi_{I}$ are eliminated by imposing continuity of fluxes on both sides of sub-interfaces.

$$
A_{L}^{3 \times 6} \Phi_{m}+B_{L}^{3 \times 3} \Phi_{I}+C_{L}^{3 \times 3} \Phi_{f}=A_{R}^{3 \times 6} \Phi_{m}+B_{R}^{3 \times 3} \Phi_{I}+C_{R}^{3 \times 3} \Phi_{f}
$$

which yields

$$
\Phi_{I}=\left(B_{L}^{3 \times 3}-B_{R}^{3 \times 3}\right)^{-1}\left(A_{R}^{3 \times 6}-A_{L}^{3 \times 6}\right) \Phi_{m}+\left(B_{L}^{3 \times 3}-B_{R}^{3 \times 3}\right)^{-1}\left(C_{R}^{3 \times 3}-C_{L}^{3 \times 3}\right) \Phi_{f}
$$


And we get the fluxes in terms of unknowns $\Phi_{m}$ and $\Phi_{f}$ only, as follows:

$$
\boldsymbol{F}=\bar{A}^{9 \times 6} \Phi_{m}+\bar{C}^{9 \times 3} \Phi_{f}
$$

where,

$$
\bar{A}^{9 \times 6}=A^{9 \times 6}+B^{9 \times 3}\left(B_{L}^{3 \times 3}-B_{R}^{3 \times 3}\right)^{-1}\left(A_{R}^{3 \times 6}-A_{L}^{3 \times 6}\right)
$$

and

$$
\bar{C}^{9 \times 3}=C^{9 \times 3}+B^{9 \times 3}\left(B_{L}^{3 \times 3}-B_{R}^{3 \times 3}\right)^{-1}\left(C_{R}^{3 \times 3}-C_{L}^{3 \times 3}\right)
$$

\subsubsection{Discontinuous pressure model}

For the second model, discontinuity in pressure is allowed across the fracture and barriers. So, at each of the sub-interfaces which are fracture cells, we specify two pressure points. Now, outward normal flux on right-edge $I_{f_{1}}$ from sub-cell of cell $m_{1}$ is computed as in (12) with;

$$
\left(\begin{array}{c}
\phi_{\xi} \\
\phi_{\eta}
\end{array}\right)=\left(\begin{array}{c}
\phi_{f_{1}}^{-}-\phi_{m_{1}} \\
\phi_{A}-\phi_{m_{1}}
\end{array}\right)
$$

Similarly, from sub-cell of cell $m_{2}$, the outward normal flux on left-edge $I_{f_{1}}$ can be computed same as in (12) with;

$$
\left(\begin{array}{c}
\phi_{\xi} \\
\phi_{\eta}
\end{array}\right)=\left(\begin{array}{c}
\phi_{B}-\phi_{m_{2}} \\
\phi_{f_{1}}^{+}-\phi_{m_{2}}
\end{array}\right)
$$

The pressures $\phi_{f_{1}}^{-}$and $\phi_{f_{1}}^{+}$are discontinuous across sub-interfaces of a fracture cell $f_{1}$ in the mesh. All the required fluxes on sub-interfaces can be formulated in the combined simple form of matrices as follows:

$$
\boldsymbol{F}=A^{9 \times 6} \Phi_{m}+B^{9 \times 9} \Phi_{I}
$$

where $\Phi_{m}=\left(\phi_{m_{1}}, \phi_{m_{2}}, \phi_{m_{3}}, \phi_{m_{4}}, \phi_{m_{5}}, \phi_{m_{6}}\right)^{T}$ are pressures associated with the matrix cells, $\Phi_{I}=\left(\phi_{A}, \phi_{B}, \phi_{C}\right.$, $\left.\phi_{f_{1}}^{-}, \phi_{f_{1}}^{+}, \phi_{f_{2}}^{-}, \phi_{f_{2}}^{+}, \phi_{f_{3}}^{-}, \phi_{f_{3}}^{+}\right)^{T}$ are pressures on the sub-interfaces, between matrix cells. As before, $\left(\phi_{A}, \phi_{B}, \phi_{C}\right)$, are eliminated by imposing continuity of fluxes across matrix sub-interfaces where fractures are not present. To eliminate discontinuous pressures on the sub-interfaces of fractures we use two transmission conditions (Robin type conditions) following [16] for each of the sub-interface. For the sub-interface $I_{f_{1}}$, the two transmission conditions are written as;

$$
\begin{aligned}
-\zeta F_{I_{f 1}}^{1}+\alpha_{f} \phi_{f_{1}}^{-} & =-(1-\zeta) F_{I_{f 1}}^{2}+\alpha_{f} \phi_{f_{1}} \\
-\zeta F_{I_{f 1}}^{2}+\alpha_{f} \phi_{f_{1}}^{+} & =-(1-\zeta) F_{I_{f 1}}^{1}+\alpha_{f} \phi_{f_{1}}
\end{aligned}
$$


where, $\phi_{f_{1}}$ is the unknown pressure associated with the involved fracture cell $f_{1}$ and specified at the centroid, follows;

$$
A_{L}^{9 \times 6} \Phi_{m}+B_{L}^{9 \times 9} \Phi_{I}=A_{R}^{9 \times 6} \Phi_{m}+B_{R}^{9 \times 9} \Phi_{I}+C_{R}^{9 \times 3} \Phi_{f}
$$

rearranging

$$
\Phi_{I}=\left(B_{L}^{9 \times 9}-B_{R}^{9 \times 9}\right)^{-1}\left(A_{R}^{9 \times 6}-A_{L}^{9 \times 6}\right) \Phi_{m}+\left(B_{L}^{9 \times 9}-B_{R}^{9 \times 9}\right)^{-1} C_{R}^{9 \times 3} \Phi_{f}
$$

where $C_{R}$ is diagonal with non-zeros corresponding to Eqs. (23), (24) and zero-rows corresponding to the matrix flux continuity conditions without fractures. Fluxes are again expressed in terms of unknowns $\Phi_{m}$ and $\Phi_{f}$ only, and are of similar form to (19). Note that, a larger local system has to be solved in (25) because of the discontinuity of fracture pressures as compared to (17) for the continuous pressure model.

Fluxes on the sub-interfaces of fractures, are retained as discontinuous for both the fracture models discussed above. The sum of the negative of these discontinuous fluxes on both sides of fracture-interfaces are the half of the transfer functions for the corresponding 1D fracture cells. At interface $I_{f_{1}}$, the sum of the negative of the discontinuous fluxes is defined as the half of the transfer function for the fracture cell $f_{1}$ :

$$
Q_{f_{1}, 1 / 2}=-F_{I_{f 1}}^{1}-F_{I_{f 1}}^{2}
$$

In the same way we can determine the half transfer functions for other fracture cells (as interfaces) involved in the cluster. As the fluxes have already been determined in terms of $\Phi_{m}$ and $\Phi_{f}$ in equation (19), so we can write the system of half transfer functions for the corresponding fracture cells in terms of $\Phi_{m}$ and $\Phi_{f}$ as

$$
\boldsymbol{Q}_{f, 1 / 2}=-\boldsymbol{F}_{L}-\boldsymbol{F}_{R}
$$


which in the above case leads to

$$
\boldsymbol{Q}_{f, 1 / 2}=\bar{D}^{3 \times 6} \Phi_{m}+\bar{E}^{3 \times 3} \Phi_{f}
$$

\subsection{Fracture-fracture fluxes}

310

315

leading to

$$
\phi_{V}=\left(B_{V}{ }^{1 \times 1}\right)^{-1} A_{V}{ }^{1 \times 3} \Phi_{f}
$$

consequently

$$
\mathcal{F}=\bar{A}^{3 \times 3} \Phi_{f}
$$

Note that, the above fracture-fracture flux formulation can be easily generalized to a cluster with any number of fracture cells. For a cluster of only two fracture cells, condition (32) is equivalent to the continuity

of fluxes between two fracture cells. 


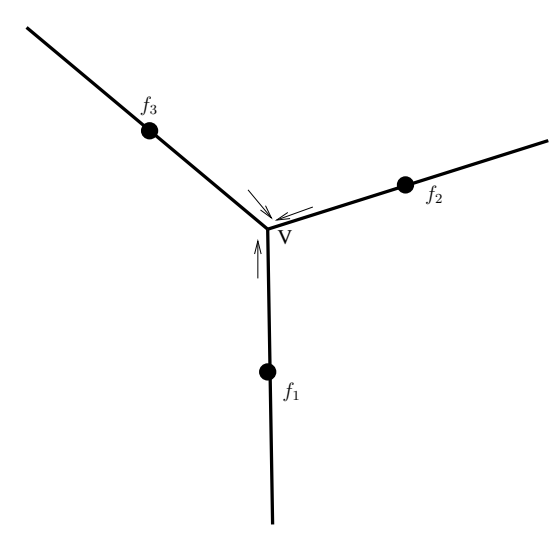

Figure 4: Cluster of fracture cells (1D cells)

\subsection{Global linear system}

The fluxes, determined in (19) and (33), and transfer function, determined in (29), complete the discrete scheme of divergence equation for every matrix cell and fracture cell.

Discrete scheme of divergence equation (10) for the matrix cell can be written as;

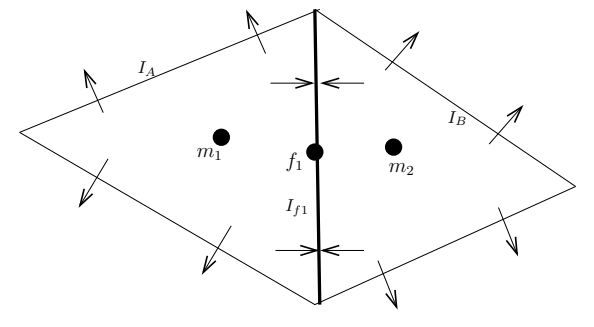

(a) All outward fluxes of the 2D matrix cells

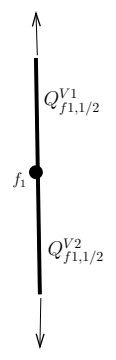

(b) All outward fluxes of the 1D fracture cell

Figure 5: Discrete scheme of divergence equation for matrix and fracture cells.

where, $F_{k}$ is the flux on half-face $k$ of the matrix cell which has $N_{e}$ number of faces and $\bar{q}_{c m}$ is the known source term. Referring to figure $5(\mathrm{a})$, triangular matrix cell has three faces (edges) so there are six outward fluxes on half-faces for the cell. For those matrix cells which are in the cluster having fracture cells, fluxes are also dependent on the fracture pressure unknowns, as defined in (19). The system of equations for discrete conservation of fluxes for all the matrix cells, using (19) and (34), can be written in system form as follows;

$$
G_{m m} \Phi_{m}+\mathcal{G}_{m f} \Phi_{f}=\overline{\boldsymbol{q}}_{c m}
$$


where $\Phi_{m}$ is unknown global pressure vector of all matrix cells, $\Phi_{f}$ is unknown global pressure vector of fracture cells and $\overline{\boldsymbol{q}}_{\boldsymbol{c}}$ is the vector of source terms corresponding to the matrix cells. $G_{m m}$ and $\mathcal{G}_{m f}$ are the linear systems corresponding to the pressure unknowns for matrix and fracture cells respectively. $\mathcal{G}_{m f}$ is the coupling of matrix pressure unknowns with the pressure unknowns for connected fracture cells because of matrix-fracture fluxes as expressed in (19). Similarly, discrete scheme of the divergence equation (11) for $1 \mathrm{D}$ fracture cell is written as;

$$
\sum_{k=1}^{2} \mathcal{F}_{k}+Q_{f}=\bar{q}_{c f} \quad \text { for every fracture cell }
$$

where $\mathcal{F}_{k}$ is the fracture-fracture flux on the face (end point) $k$ of $1 \mathrm{D}$ fracture cell. For 1D fracture cells, in 2D problem, there will be two faces (end points) for every fracture cell as shown in figure 5(b). $Q_{f}$ and $\bar{q}_{c f}$ are the transfer function and any known source term respectively for the corresponding fracture cell. Fracturefracture fluxes are determined in (33). Because each fracture cell is common to two neighboring clusters of matrix cells, so the total transfer function $Q_{f}$ is determined by the addition of half transfer functions determined as (29), from corresponding clusters, in terms of matrix and fracture pressure unknowns. The system of equations of discrete conservation of fluxes for all the fracture cells, using (33) and (36), can be written as;

$$
G_{f f} \Phi_{f}+\boldsymbol{Q}_{f}=\overline{\boldsymbol{q}}_{c f}
$$

where $G_{f f}$ corresponds to the fracture-fracture fluxes, $\overline{\boldsymbol{q}}_{\boldsymbol{c} f}$ is the vector of known source terms for the fracture cells and the $\boldsymbol{Q}_{f}$ is the vector of transfer functions for fracture cells. Using (29), $\boldsymbol{Q}_{f}$ is replaced in equation (37) and we get:

$$
\begin{aligned}
G_{f f} \Phi_{f}+\mathcal{G}_{T m} \Phi_{m}+\mathcal{G}_{T f} \Phi_{f} & =\overline{\boldsymbol{q}}_{c f} \\
\text { or } \quad\left(G_{f f}+\mathcal{G}_{T f}\right) \Phi_{f}+\mathcal{G}_{T m} \Phi_{m} & =\overline{\boldsymbol{q}}_{c f}
\end{aligned}
$$

where $\mathcal{G}_{T m}$ and $\mathcal{G}_{T f}$ correspond to transfer function matrix coefficients for the fracture cells. $\mathcal{G}_{T m}$ is the coupling between pressure unknowns for matrix and fracture cells.

Thus we have to solve two coupled systems from, equation (35) and equation (38), for unknown pressures in matrix and fracture cells respectively. These can be written in coupled linear system form as follows:

$$
\left(\begin{array}{cc}
G_{m m} & \mathcal{G}_{m f} \\
\mathcal{G}_{T m} & G_{f f}+\mathcal{G}_{T f}
\end{array}\right)\left(\begin{array}{c}
\Phi_{m} \\
\Phi_{f}
\end{array}\right)=\left(\begin{array}{c}
\overline{\boldsymbol{q}}_{c m} \\
\overline{\boldsymbol{q}}_{c f}
\end{array}\right)
$$


Using simplified notation of $\bar{G}_{f f}=G_{f f}+\mathcal{G}_{T f}$, we have;

$$
\left(\begin{array}{cc}
G_{m m} & \mathcal{G}_{m f} \\
\mathcal{G}_{T m} & \bar{G}_{f f}
\end{array}\right)\left(\begin{array}{c}
\Phi_{m} \\
\Phi_{f}
\end{array}\right)=\left(\begin{array}{c}
\overline{\boldsymbol{q}}_{c m} \\
\overline{\boldsymbol{q}}_{c f}
\end{array}\right)
$$

The above coupled system, (39) can be solved by iterative solution methods for matrix and fracture pressures. It is noted that a smaller linear system is solved locally, as in (17), for every cluster as compared to the hybrid-grid method because of lower-dimensional fracture cells. Matrix-matrix fluxes and matrix-fracture

\section{Transport model}

An explicit first order upwind (upstream weighting) method is used for transport of tracer by the fluid through fractured porous medium. We treat the intersecting fractures in the same way as treated in 2] and 18]. The discrete transport equation for the fracture cell can be written as ;

$$
\varphi \frac{c^{n+1}-c^{n}}{\delta t} A_{f}+\sum_{k=1}^{2} \mathcal{F}_{k} c_{f o}-F_{m_{1}} c_{1}-F_{m_{2}} c_{2}=q_{c f} A_{f} \quad \text { for time step } n
$$


where, $c$ is the concentration of the tracer in fracture cell of area $A_{f}, \mathcal{F}_{k}$ are the fracture-fracture fluxes, $F_{m 1}$ and $F_{m 2}$ are the matrix-fracture fluxes, outward normal to neighboring matrix cells $m_{1}$ and $m_{2}$, as computed in previous section (expressions (12) and (14)). $c_{1}$ and $c_{2}$ are taken as concentration of fracture cell or neighboring matrix cell considering the upstream direction of fluxes i.e., into the fracture or out of the fracture. $c_{f o}$ is the concentration at the intersection point between the intersecting fracture cells; depicted by $V$ in figure 4 . We assume that the flow is fast in the fractures such that there is no accumulation of mass at the intersection point. If there are $p$ intersecting fractures meeting at the point and there are $l$ fluxes going into the intersection point then we can compute $c_{f o}$ by the following condition;

$$
\sum_{k=1}^{l} \mathcal{F}_{k} c_{k}=c_{f o} \sum_{k=1}^{p-l} \mathcal{F}_{k}
$$

In this way we do not need to include the small intermediate cell explicitly, as in equi-dimensional model, in overall computations and avoid the restriction of a low CFL condition that would result from inclusion of the intermediate cell.

\section{Numerical results}

Numerical results are presented in this section to assess the lower-dimensional fracture models. First of all we show the convergence test of our coupled CVD-MPFA formulation. Then we expand on the difference in the two variations of lower-dimensional fracture model. Moreover, we assess the accuracy of lowerdimensional fracture model for a challenging problem and observe the effect of value of $\zeta$ parameter used in the formulation. Then, we compare the pressure and tracer transport solutions given by lower-dimensional model, hybrid-grid and equi-dimensional fracture models on unstructured meshes. We also present the comparison of the computational cost for the lower-dimensional and the equi-dimensional fracture models. A transient pressure simulation is presented in the last sub-section for the highly fractured medium.

\subsection{Convergence test}

First of all we perform the convergence tests for a domain with single fracture as used in [17, 18]. Figure 6 shows the domain with single fracture of aperture $a$. Permeability of the domain is piecewise constant in matrix and fracture:

$$
\boldsymbol{K}(x, y)= \begin{cases}\boldsymbol{K}_{m}(x, y) & (x, y) \in \Omega_{m} \\ k_{f} \boldsymbol{K}_{m}(x, y) & (x, y) \in \Omega_{f}\end{cases}
$$

where $\boldsymbol{K}_{m}=\left(\begin{array}{ll}1 & 0 \\ 0 & 1\end{array}\right)$ and $k_{f}$ is the permeability ratio of fracture to that of matrix. Exact pressure for the domain (6) is given by : 


$$
\phi(x, y)= \begin{cases}k_{f} \cos (x) \cosh (y)+\left(1-k_{f}\right) \cos (x) \cosh (a / 2) & (x, y) \in \Omega_{m} \\ \cos (x) \cosh (y) & (x, y) \in \Omega_{f}\end{cases}
$$

for the source:

$$
q_{c}(x, y)= \begin{cases}\left(1-k_{f}\right) \cos (x) \cosh (a / 2) & (x, y) \in \Omega_{m} \\ 0 & (x, y) \in \Omega_{f}\end{cases}
$$

Dirichlet boundary conditions are imposed on all the boundaries of the concerned domain. We discretize the

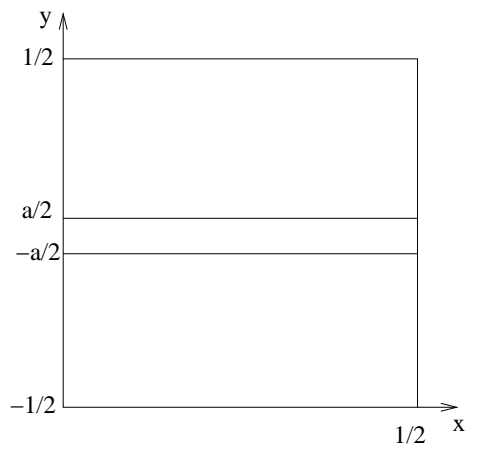

Figure 6: Square domain with a single fracture, of aperture $a$, in the middle.

domain into $N \times N$ regular quadrilateral elements where fracture is represented by the interfaces between the elements. We compute the normalized error norms for pressure as defined in [18]. Normalized error norms of pressure are plotted against the aperture to cell length ratio $a / L_{h}$ for a series of $N \times N$ elements where $N=10,20, \ldots, 100$. For comparison, convergence plots of the lower-dimensional(1D) fracture model (broken lines) and equi-dimensional(2D) fracture model (continuous lines) are given in figure 7 for different values of aperture $a$ and permeability contrast $k_{f}$. When plots for the two models merge, only one plot appears in the figure. Note that, we have checked the convergence of the both variations of lower-dimensional model that are continuous pressure and discontinuous pressure across fracture, with different values of parameter $\zeta=\{0.51,2.0 / 3.0,3.0 / 4.0,1.0\}$, and we find no considerable difference in the errors of the solutions given by different models. Referring to convergence plots, when the aperture is higher $\left(a=10^{-3}\right)$, the lowerdimensional fracture model diverges (at $a / L_{h}=0.02$ ), from the convergence plot of the equi-dimensional model, for the cases of high conductive fracture $\left(k_{f}=10^{4}\right)$ and equal permeable fracture $\left(k_{f}=1\right)$ as shown by the convergence plot $7(\mathrm{a})$ The largest discrepancy, between the two models, is shown for a homogeneous case, $\left(k_{f}=1\right)$, when the aperture value is high $\left(a=10^{-3}\right)$. But, lower-dimensional fracture model is accurate for the low permeable fracture for all cases of aperture values (the pressure field is symmetric about the fracture). Also, lower-dimensional fracture model shows better convergence for higher permeable fracture and equal permeable fracture when aperture is low as shown in plots $7(\mathrm{~b})$ and $7(\mathrm{c})$. This shows that the lower-dimensional fracture model is accurate when $a / L_{h}<<1$ for highly conductive fractures. Identical convergence has been obtained by the hybrid-grid method in [18]. Note that, if precise geometry is used during computations with the hybrid-grid method, that is the geometry of neighboring matrix is adjusted 


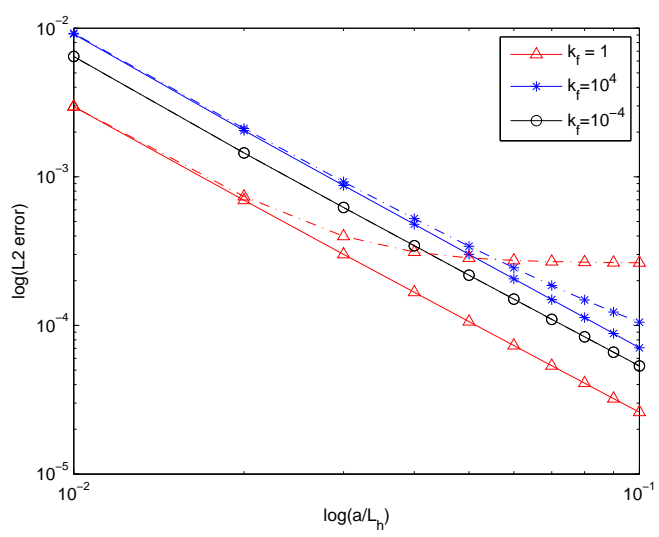

(a) aperture, $a=10^{-3}$

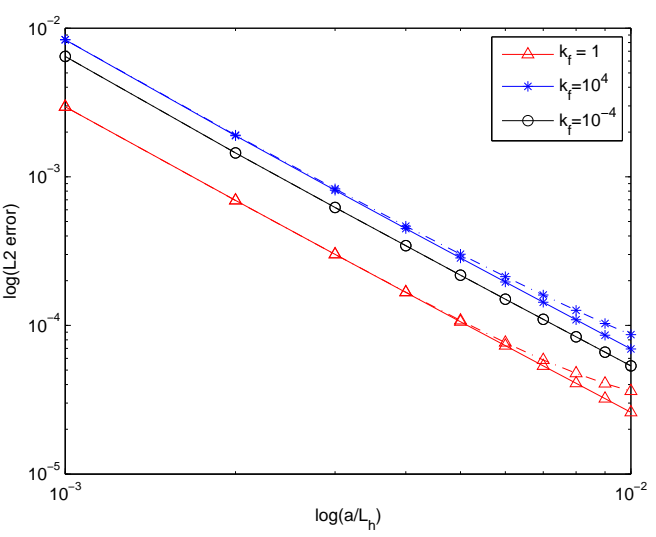

(b) aperture, $a=10^{-4}$

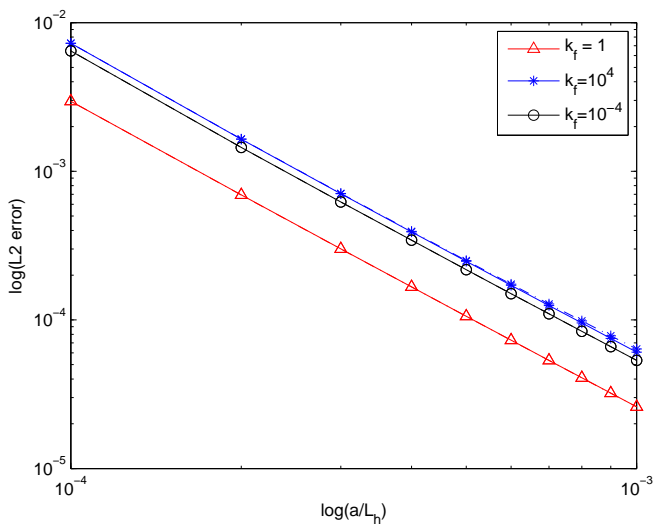

(c) aperture, $a=10^{-5}$

Figure 7: Normalized error vs $a / L_{h}$ for different aperture values and for different permeability contrast ratio $k_{f}$. $L_{h}$ is the cell length of the grid. Continuous lines show convergence for the equi-dimensional model while broken lines show convergence of the lower-dimensional fracture model.

when fractures are expanded in computational domain, then the convergence of the hybrid-grid method

would be the same as that of the equi-dimensional model for this case (non-intersecting fractures).

\subsection{Single Fracture; Isotropic permeability}

We solve two cases involving a single fracture domain with different permeability and boundary conditions, as presented in [16], by lower-dimensional (1D) fracture continuous pressure model and discontinuous pressure model to show the difference in two models. The aperture of the fractures in both cases is $a=0.01 \mathrm{~m}$. The lengths of the domain in $x$ and $y$ directions are $L_{x}=2 m$ and $L_{y}=1 m$ respectively. The figure 8 shows the boundary conditions for the two cases.

Case 1: Permeability of whole fracture is higher than that of matrix; $\boldsymbol{K}_{f_{1}}=\boldsymbol{K}_{f_{2}}=100 \boldsymbol{K}_{m} ; \boldsymbol{K}_{m}=\boldsymbol{I} m D$. Dirichlet boundary condition is applied for fracture ends.

Case 2: Permeability of the middle part of fracture is lower than that of matrix; $\boldsymbol{K}_{f_{2}}=0.002 \boldsymbol{K}_{m}$. The permeability of rest of the fracture is same as that of matrix $\boldsymbol{K}_{f_{1}}=\boldsymbol{K}_{m}=\boldsymbol{I} m D$. Neumann boundary condition is applied for the fracture ends. As shown in figure 9 the continuous pressure model gives the same 


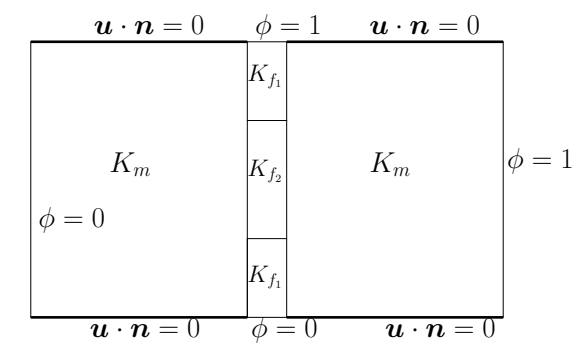

(a) Case 1

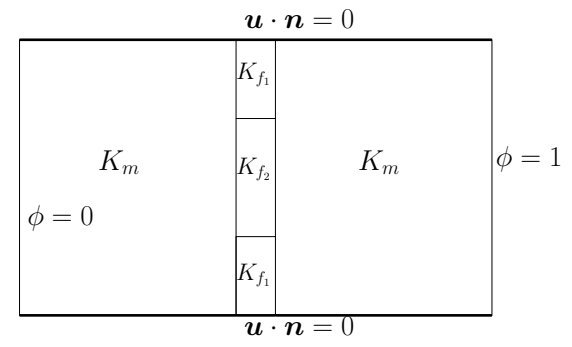

(b) Case 2

Figure 8: Domain and boundary conditions for single fracture tests

results for case 1 as also given by the discontinuous pressure model where fracture has high permeability in normal and tangential directions. The pressure variation across fracture is very small and can efficiently be modeled by the 1D fracture continuous pressure model. For case 2, when the fracture permeability is low $\left(\boldsymbol{K}_{f_{2}}=0.002 \boldsymbol{K}_{m}\right)$, continuous pressure model does not show the pressure jump across fracture as compared to discontinuous model which yields result in agreement with result shown in [16]. The continuous pressure model for low permeability shows good convergence c.f. figure 7 in previous sub-section and we note that in this problem the pressure field is symmetric about the fracture. Results, in figure 9 show that the continuous pressure model is efficient for the thin fractures which have high permeability in the normal direction, without adding extra degrees of freedom across fracture.

\subsection{Single fracture; Anisotropic discontinuous permeabilities and BCs}

In this section we assess the accuracy of the 1D fracture discontinuous pressure model for anisotropic single fracture in the domain as shown in figure $8(\mathrm{a})$ and observe the influence of different values of parameter $\zeta$. Permeability of the rock-matrix is identity $\boldsymbol{K}_{m}=\boldsymbol{I} m D$ and permeability of fracture is anisotropic and discontinuous $\boldsymbol{K}_{\boldsymbol{f} \mathbf{1}}=\left(\begin{array}{cc}1 / k_{f} & 0.0 \\ 0.0 & k_{f}\end{array}\right) m D$ and $\boldsymbol{K}_{\boldsymbol{f} \mathbf{2}}=\left(\begin{array}{cc}k_{f} & 0.0 \\ 0.0 & 1 / k_{f}\end{array}\right) m D$ where $k_{f}>1.0$. So, fluid cannot flow along the middle part of the fracture but can cross it. The flow behavior is opposite in the lower and upper parts of the fracture. Dirichlet boundary condition is applied at the ends of fracture. We solve the problem by the lower-dimensional model and assess its accuracy with respect to the parameter $\zeta$ of model and fracture attributes $\left(k_{f}\right.$, aperture $a$ in $\left.m\right)$. As we do not have an analytical solution of this problem, so we solve the problem by the equi-dimensional method (CVD-MPFA, $q=1.0$ ) using much refined mesh which is taken as our reference solution. Uniform structured quadrilateral mesh is used for this test case. The cell size of the mesh for the reference solution is $L_{h}^{*} \approx 1.0 / 320$ and $2 \mathrm{D}$ fracture cell size is $L_{h, f}^{*}=a / 3$ in normal direction. The mesh cell size for the 1D fracture model is $L_{h}=1.0 / 160$. Figure 10 shows the reference pressure solution and the pressure solution given by the lower-dimensional fracture model with different values of parameter $\zeta$ for the fracture aperture $a=1 e-2$ and $k_{f}=200$. For $\zeta=2.0 / 3.0$ the behavior of the solution is the same as that of the reference solution. The solutions produced by $\zeta=0.51$ and $\zeta=0.49$ have spurious oscillations close to the fracture and violate the discrete maximum principle(DMP) so these values 


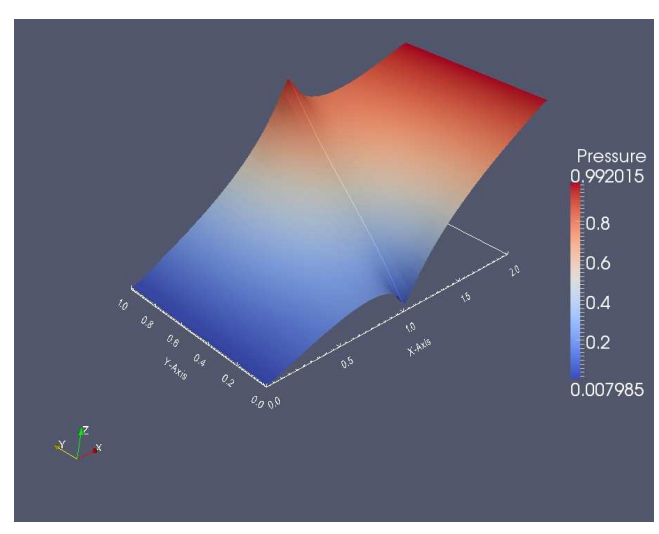

(a) Case 1, Discont. pressure model

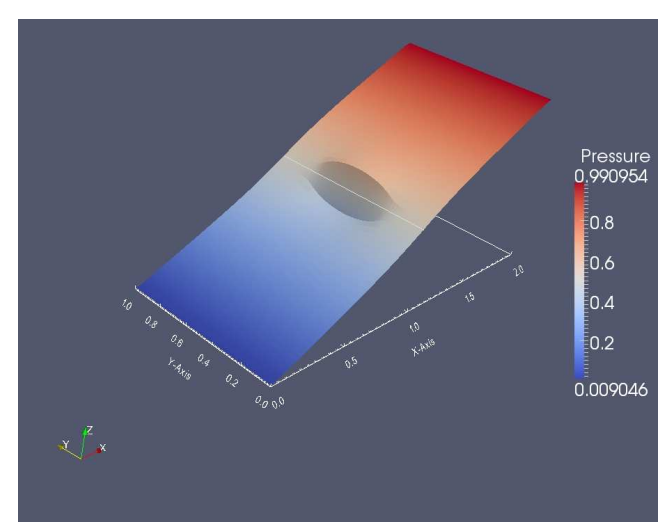

(c) Case 2, Discont. pressure model

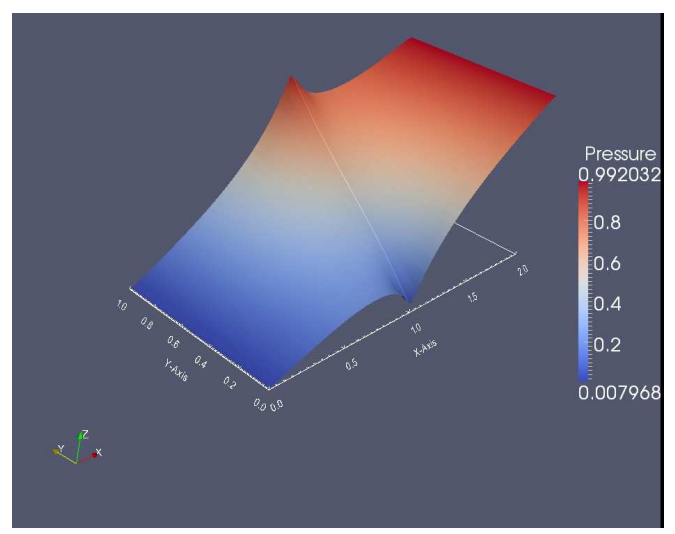

(b) Case 1, Cont. pressure model

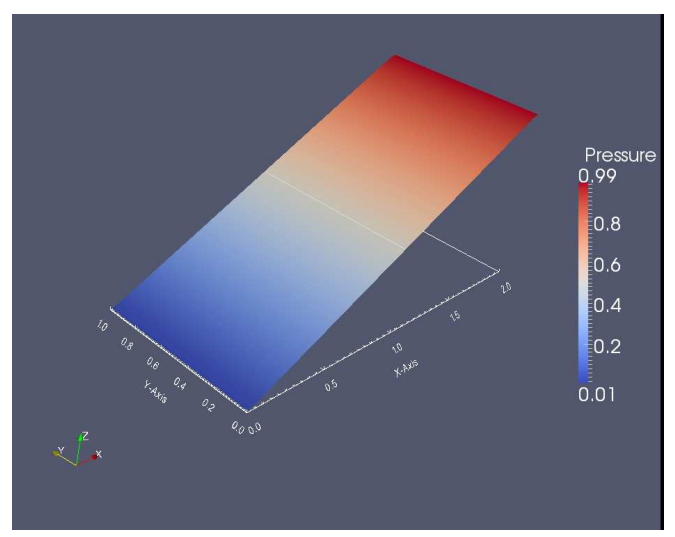

(d) Case 2, Cont. pressure model

Figure 9: Simulation results of single fracture domain with different permeability and boundary conditions given by 1D fracture continuous and discontinuous pressure models

of $\zeta$ should be avoided for high aperture values. Same behavior is observed in [16] in the context of mixed finite-element method. Pressure solutions for the fracture aperture $a=5 e-4$ and $k_{f}=200$ are shown in figure 11. Note that there is no violation of DMP for very low aperture $a=5 e-4$, for $\zeta=0.51$ and $\zeta=0.49$. Figure 12 shows the reference solution and 1D fracture model $(\zeta=2.0 / 3.0)$ results for $k_{f}=20$ and for high aperture $a=1 e-2$ and low aperture $a=5 e-4$. There is a local pressure behavior close to the fracture when the aperture is very low.

The accuracy of the 1D fracture model, with different values of parameter $\zeta$, and hybrid-grid method with respect to the fracture aperture $a$ and for $k_{f}$ is shown in the figure 13 The relative $L^{2}$ error of the solution is not affected by the change of aperture if $k_{f} * a=1.0$ as shown in the figure 13(a) but the solution behavior changes by the change of parameter $\zeta$ of the model and we get the best accuracy for $\zeta=2.0 / 3.0$. For $\zeta>1.0$ a large error in the solution is obtained and we note that this corresponds to an extrapolation out of limits. Figure 13(b) shows the relative $L^{2}$ error versus fracture aperture for high permeability contrast $k_{f}=200$. For this case the relative $L^{2}$ error increases with the increase of the aperture. The relative $L^{2}$ error versus aperture plot for low permeability contrast $k_{f}=20$ is shown in figure 13(c). 


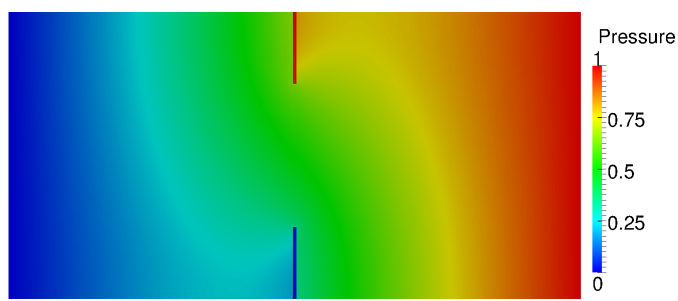

(a) Reference solution.

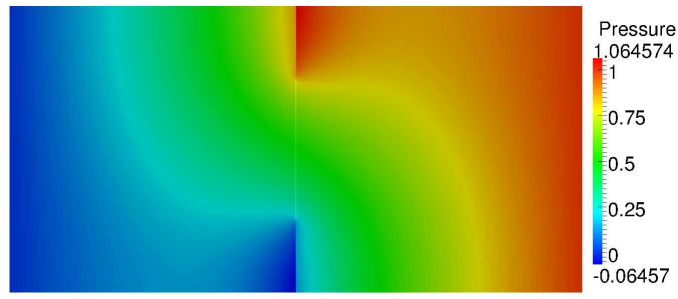

(c) $\zeta=0.51$

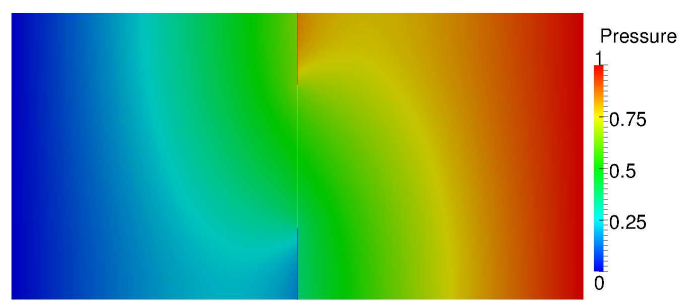

(b) $\zeta=2.0 / 3.0$

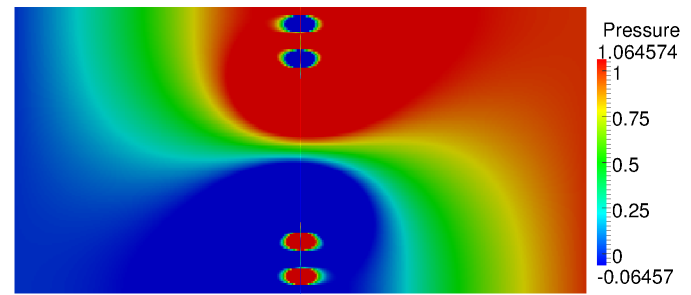

(d) $\zeta=0.49$

Figure 10: Reference pressure plot and the lower-dimensional fracture solutions for $a=1 e-2, k_{f}=200$.

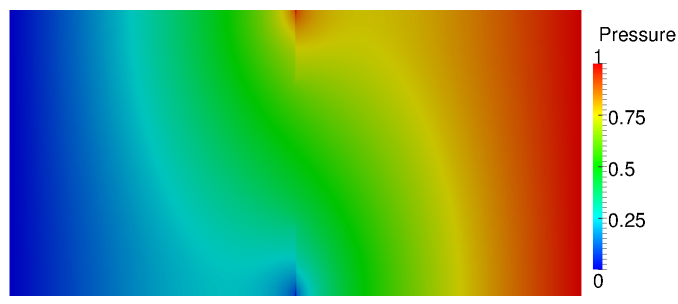

(a) Reference solution.

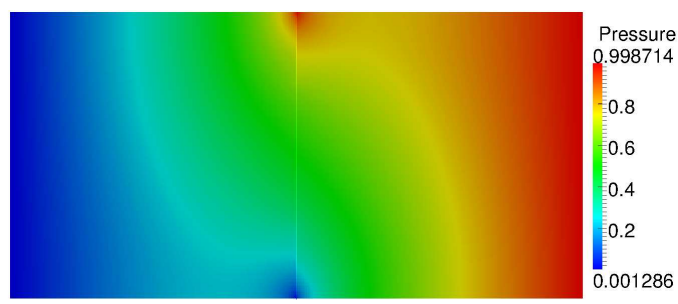

(c) $\zeta=0.51$

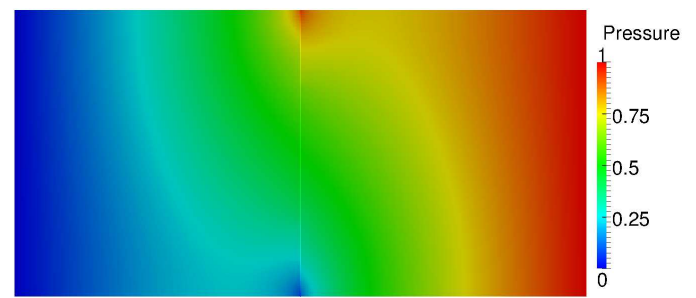

(b) $\zeta=2.0 / 3.0$

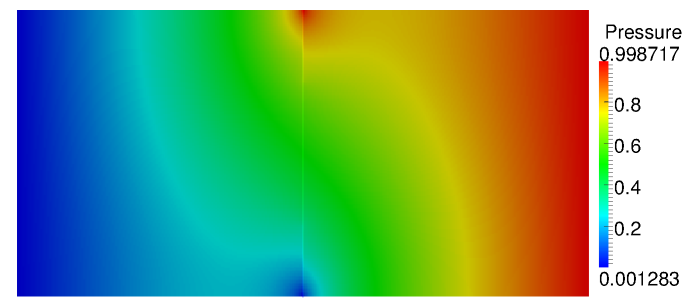

(d) $\zeta=0.49$

Figure 11: Reference pressure plot and the lower-dimensional fracture solutions for $a=5 e-4, k_{f}=200$.

For the cases discussed here, $\zeta=2.0 / 3.0$ yields best accuracy of the solution with $1 \mathrm{D}$ fracture model.

490 better than $\zeta=2.0 / 3.0$.

\subsection{Comparison of transport solution with the 2D fracture model}

We now solve the transport problems using different fracture models as discussed above. In the first part, we solve the problem for the domain with a single fracture and second part deals with the domain 


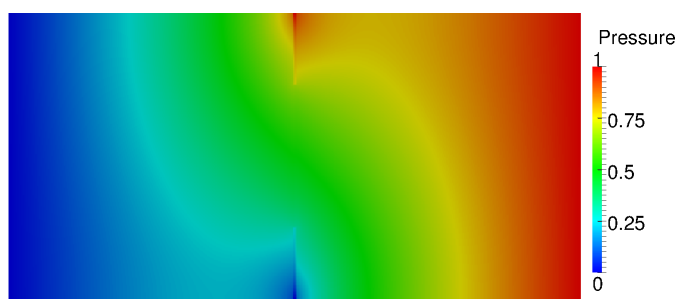

(a) Reference solution $a=1 e-2$

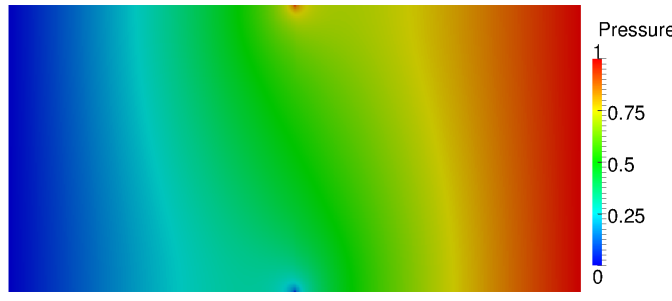

(c) Reference solution $a=5 e-4$

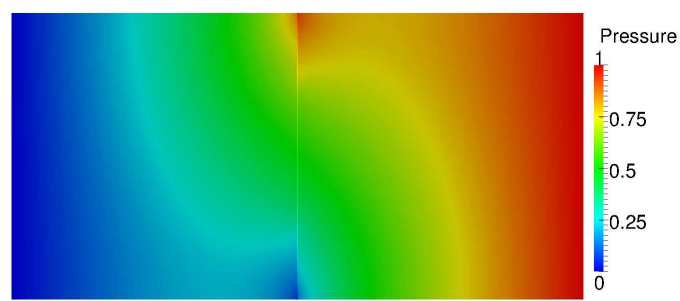

(b) $a=1 e-2, \zeta=2.0 / 3.0$

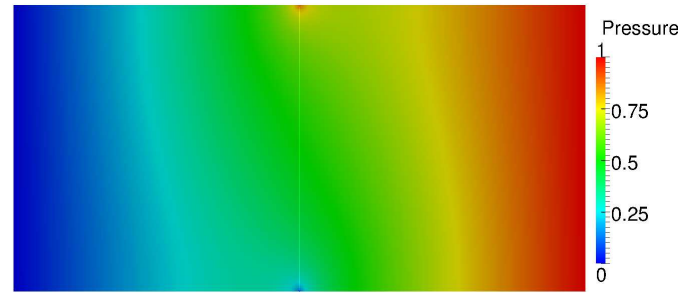

(d) $a=5 e-4, \zeta=2.0 / 3.0$

Figure 12: Reference pressure plot and the lower-dimensional fracture solutions for $k_{f}=20$ and $a=1 e-2 \& a=5 e-4$

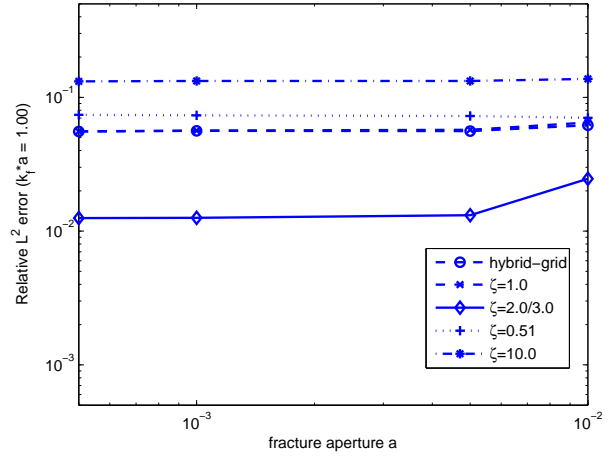

(a) $k_{f} * a=1.0$

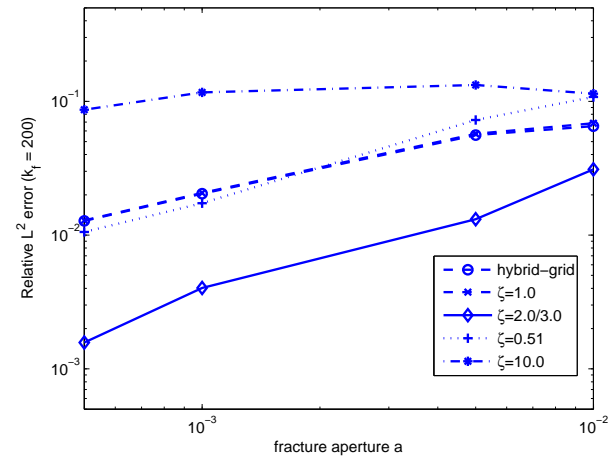

(b) $k_{f}=200$

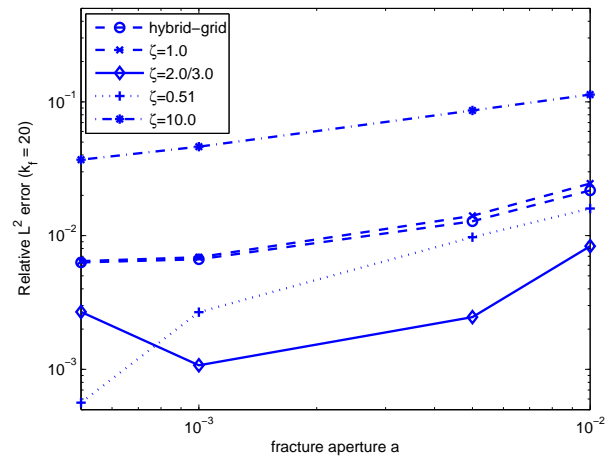

(c) $k_{f}=20$

Figure 13: Relative $L^{2}$ error variation versus the fracture aperture. For 1D fracture model mesh has $320 \times 160$ quadrilaterals. Cell length, $L_{h}=1 / 160$

ii) hybrid-grid method and iii) equi-dimensional model (2D fracture) where fractures are gridded explicitly 
in the physical mesh. The same time step-size has been used for all three fracture models. We solve the problems with both variations of the $1 \mathrm{D}$ fracture model that are with continuous pressure and discontinuous pressure across the fracture and show contours and plots of the solutions by discontinuous pressure model as both give similar solutions for permeable fractures. We will refer to the discontinuous pressure model by $1 D$ fracture and continuous pressure model by $1 D$ fracture cont. We compare the results of concentration variation with time at the producer. In $2 \mathrm{D}$ fracture meshes, the whole domain is discretized by the triangular cells including the thin fracture. Using $q=2 / 3$ ensures the scheme is SPD. Moreover, the mesh is refined very close to the fracture to keep the quality of the mesh uniform within $2 \mathrm{D}$ fractures. For the 1D fracture model, the fracture is treated as an internal boundary constraint and discretized along the specified edges of the 2D triangular cells. Grids for the tests in the following sub-sections have been generated by using the Triangle 41] unstructured mesh generator.

\subsubsection{Single fracture}

We solve the problem for a domain with a single fracture with aperture $a=10^{-3} \mathrm{~m}$ and the permeability ratio of the fracture to matrix is $k_{f}=10^{4}$. We consider two cases of rock-matrix permeability a) isotropic identity tensor $\boldsymbol{K}_{m}=\boldsymbol{I} m D$ and b) anisotropic tensor $\boldsymbol{K}_{\boldsymbol{m}}=\left(\begin{array}{cc}7.75 & 3.8971 \\ 3.8971 & 3.25\end{array}\right) m D$ (anisotropy ratio of 10 : 1 at angle $30^{\circ}$ ) and the fracture permeability is $\boldsymbol{K}_{f}=k_{f} \boldsymbol{K}_{m}$. This domain has been used in [42] for multiphase flow problem using the FEM method. Length and height of the domain is $L_{x}=L_{y}=1 \mathrm{~m}$. The meshes are shown in the figure 14 Zero-flux Neumann boundary condition is imposed on the whole external boundary of the domain. Fluid is injected through an injector I (with rate 0.3171 PV per year) and zero-pressure is specified at the producer. The concentration of the tracer is determined by solving the transport equation for each time step. Pressure contours given by the three fracture models for isotropic and anisotropic case are given in figure 15 and figure 17. Tracer concentration contours at pore volume injected $(P V I)=1.2716$ are shown in figure 16 and figure 18. Note that no considerable discrepancy has been found in the results given by $1 \mathrm{D}$ fracture model with different values of $\zeta$. In this and the following subsections, we only show results for $\zeta=2.0 / 3.0$. Behavior of respective pressure and tracer concentration solution using the hybrid-grid and $1 \mathrm{D}$ fracture model approaches the solution by $2 \mathrm{D}$ fracture model. It can be seen that fluid tends to flow faster along the fracture for isotropic case but in the second case, anisotropic permeability field overwhelms the presence of conductive fracture with the specified boundary conditions. Tracer concentration at the producer is recorded for every time step. The variation of concentration at producer with time for three fracture models are shown in figure 19 for cases of isotropic and anisotropic permeability. Concentration values at $P V I=1.9026$ are also given in table 1 These plots show that there is very little discrepancy between the solutions of hybrid-grid and 1D fracture models for both the cases. For isotropic case, both the models yield results that are in excellent agreement with the 2D fracture model results. In this case we conclude that lower-dimensional modeling yields comparable results to that of the hybrid-grid and 2D fracture models for thin highly conductive fractures. 


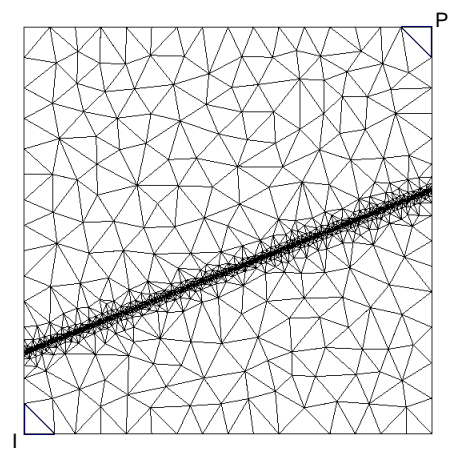

(a) aperture $=10^{-3}, 4159$ cells

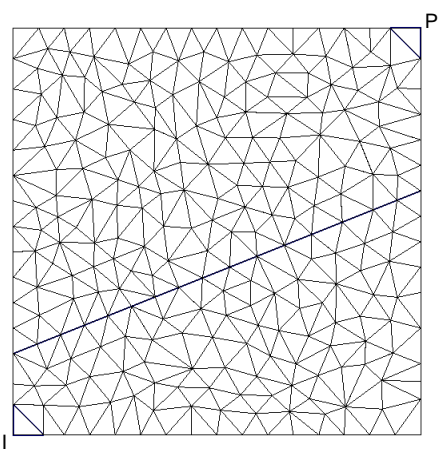

(b) 456 cells $+161 \mathrm{D}$ fracture cells

Figure 14: a) Explicit grid representation of fracture and b) mixed-dimensional grid for domain with single fracture. Injector is marked by I and producer is marked by $\mathrm{P}$.

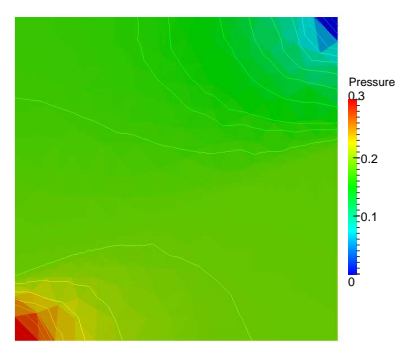

(a) 2D fracture model

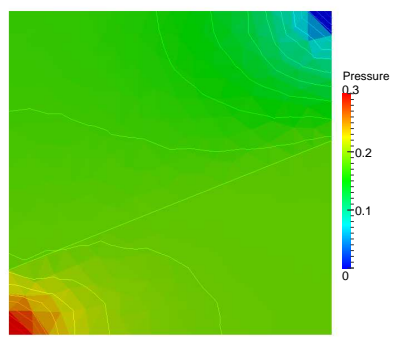

(b) Hybrid-grid model

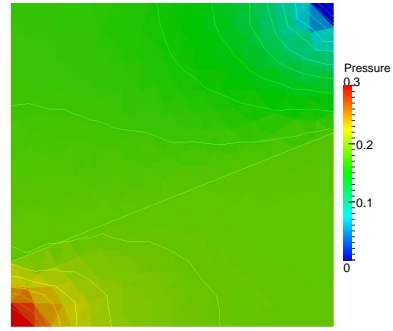

(c) $1 \mathrm{D}$ fracture model $(\zeta=$ $2 / 3)$

Figure 15: Pressure contours by three fracture models for single fracture domain with isotropic permeability tensor and $a=$ $10^{-3} \mathrm{~m}, k_{f}=10^{4}$.

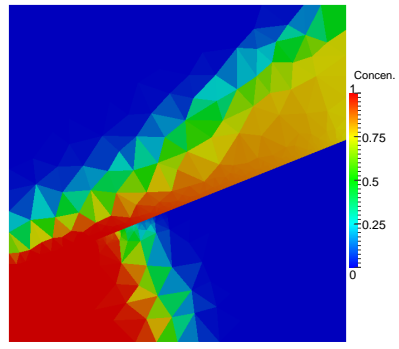

(a) $2 \mathrm{D}$ fracture

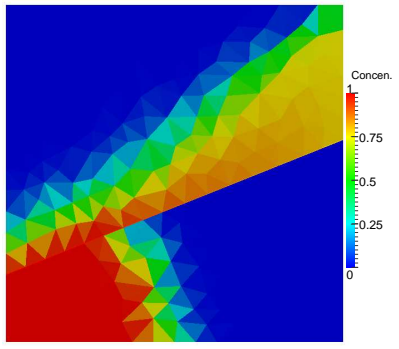

(b) Hybrid-grid

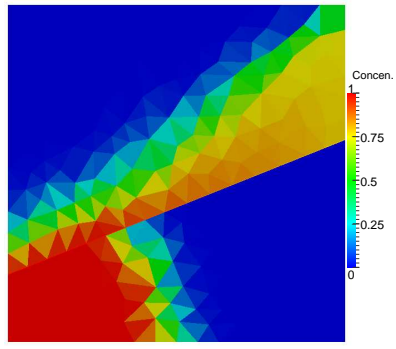

(c) $1 \mathrm{D}$ fracture

Figure 16: Tracer concentration contours at $P V I=1.2716$ by three fracture models for single fracture domain with isotropic permeability and $a=10^{-3} m, k_{f}=10^{4}$.

\subsubsection{Intersecting fractures}

In this section we consider the problem of domain with two intersecting fractures. The results are obtained by i) lower-dimensional fracture model (1D fracture), ii) hybrid-grid method and iii) equi-dimensional model (2D fracture). Length and height of the domain is $L_{x}=L_{y}=1 \mathrm{~m}$. The meshes with explicit 2D 


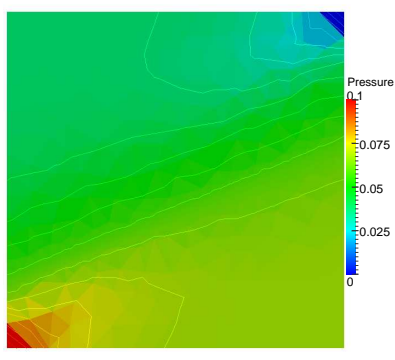

(a) $2 \mathrm{D}$ fracture

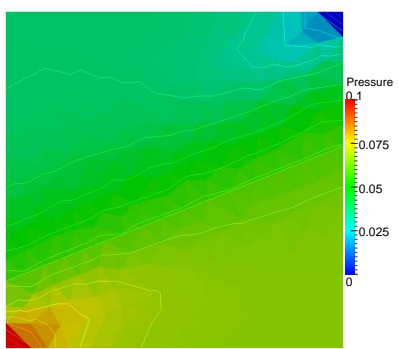

(b) Hybrid-grid

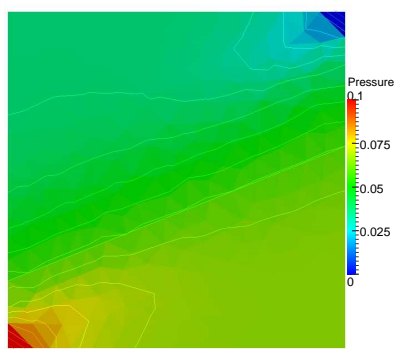

(c) $1 \mathrm{D}$ fracture

Figure 17: Pressure contours by three fracture modeling for single fracture domain with anisotropic permeability tensor and $a=10^{-3} m, k_{f}=10^{4}$.

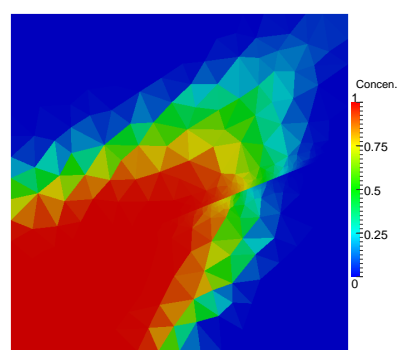

(a) $2 \mathrm{D}$ fracture

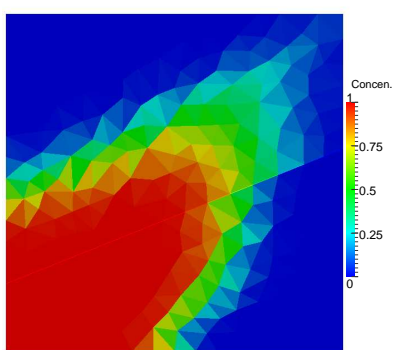

(b) Hybrid-grid

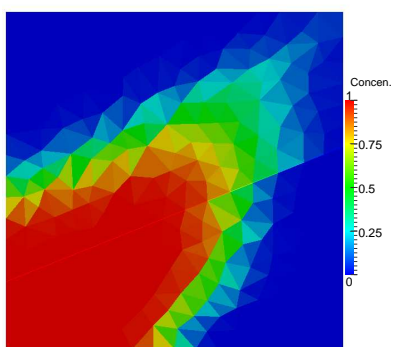

(c) $1 \mathrm{D}$ fracture

Figure 18: Tracer concentration contours at $P V I=1.2716$ by three fracture models for single fracture domain with anisotropic permeability tensor and $a=10^{-3} m, k_{f}=10^{4}$.

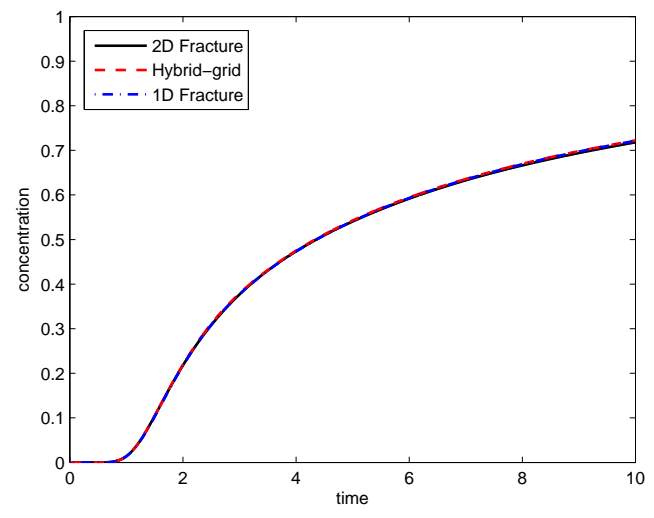

(a) Isotropic case

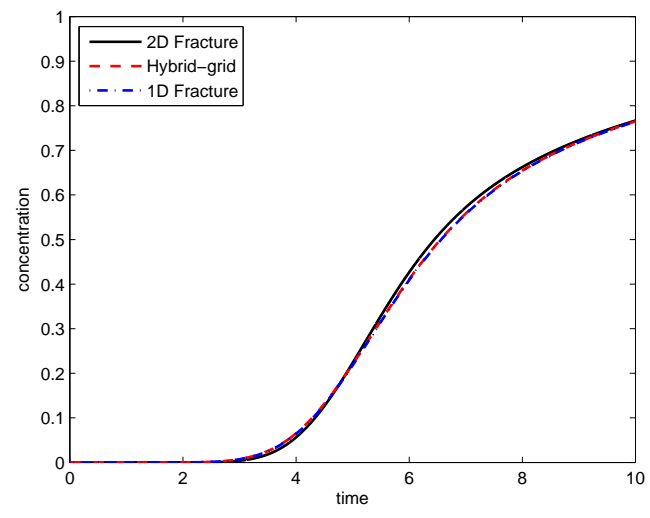

(b) Anisotropic case

Figure 19: Plots of tracer concentration at producer w.r.t time for domain of single fracture with $a=10^{-3} \mathrm{~m}, k_{f}=10^{4}$

fracture and $1 \mathrm{D}$ fracture representations are shown in figure 20, The aperture of both the fractures is $10^{-3} \mathrm{~m}$ and two different permeability ratios are taken into consideration i.e., $k_{f}=10^{4}$ and $k_{f}=10^{6}$. Both fractures have the same permeability. Matrix permeability is defined by the identity tensor; $\boldsymbol{K}_{m}=\boldsymbol{I} m D$ for the first case and by anisotropic permeability of ratio $10: 1$ at angle $30^{\circ}$ for second case. Fracture permeability is 


\begin{tabular}{l|c|c} 
Fracture model & Isotropic case & Anisotropic case \\
\hline 2D fracture & 0.5916995 & 0.4264542 \\
Hybrid-grid & 0.5941573 & 0.4107767 \\
1D fracture & 0.5929003 & 0.4088482 \\
1D fracture cont. & 0.5929003 & 0.4088482
\end{tabular}

Table 1: Tracer concentration at producer at $P V I=1.9026$ for isotropic and anisotropic cases with single fracture of $a=10^{-3} m$, $k_{f}=10^{4}$

$\boldsymbol{K}_{f}=k_{f} \boldsymbol{K}_{m}$ for both isotropic and anisotropic cases. Zero-flux Neumann condition is imposed on the whole external boundary of the domain. Fluid is injected through an injector I (with rate $0.6342 \mathrm{PV}$ per year) and zero-pressure is specified at the producer. Respective pressure and tracer concentration fields, produced by the three models are shown in figure 21 and figure 22. with $k_{f}=10^{4}$, for the cases of isotropic and anisotropic permeabilities respectively. Solution contours are similar for $k_{f}=10^{6}$. Tracer concentration at

the producer is recorded for each time step. The variation of tracer concentration at the producer with time computed by the three methods are shown in figures 23 and 24. Table 2 shows the concentration values at $P V I=3.8052$. The concentration plots show that the $1 \mathrm{D}$ fracture model and hybrid-grid model yield results that are in excellent agreement with those of the 2D fracture model. Both the models treat the intersecting fractures with the same assumptions of no accumulation and continuity of pressure at the intersection point. There is a discrepancy between concentrations given by the 1D fracture model, hybrid-grid model and the equi-dimensional model which is higher for $k_{f}=10^{4}$ in contrast to the case of $k_{f}=10^{6}$. The discrepancy in the concentration results is less for the anisotropic case than for the isotropic case. These results indicate that the 1D fracture model and hybrid-grid model have similar accuracy for the fully immersed intersecting conductive fractures when compared to the explicitly modeled fracture solution. Both models give accurate tracer transport results for higher permeable intersecting fractures because of the similarity in the treatment and assumptions for the intersecting fractures to avoid the explicit representation of small intermediate cell which is included in equi-dimensional model. Moreover, as shown in table 2 1D fracture model with continuous pressure across fracture gives the same results as obtained by the discontinuous pressure model for the conductive fractures. We note that discontinuous fracture pressure approximation is only required when the fracture with low-permeability, in the normal direction, is encountered in the domain.

\begin{tabular}{l|c|c||c|c} 
& \multicolumn{2}{|c}{$\boldsymbol{k}_{\boldsymbol{f}}=\mathbf{1 0}^{\mathbf{4}}$} & \multicolumn{2}{c}{$\boldsymbol{k}_{\boldsymbol{f}}=\mathbf{1 0}^{\mathbf{6}}$} \\
Fracture model & Isotropic case & Anisotropic case & Isotropic case & Anisotropic case \\
\hline 2D fracture & 0.7262379 & 0.8355235 & 0.7409754 & 0.8375609 \\
Hybrid-grid & 0.7071900 & 0.8306804 & 0.7366143 & 0.8361955 \\
1D fracture & 0.7057403 & 0.8324591 & 0.7351484 & 0.8356579 \\
1D fracture cont. & 0.7057403 & 0.8324591 & 0.7351484 & 0.8356579
\end{tabular}

Table 2: Tracer concentration at producer at $P V I=3.8052$ for isotropic and anisotropic cases with intersecting fractures of $a=10^{-3} m$ and $k_{f}=10^{4} \& k_{f}=10^{6}$ 


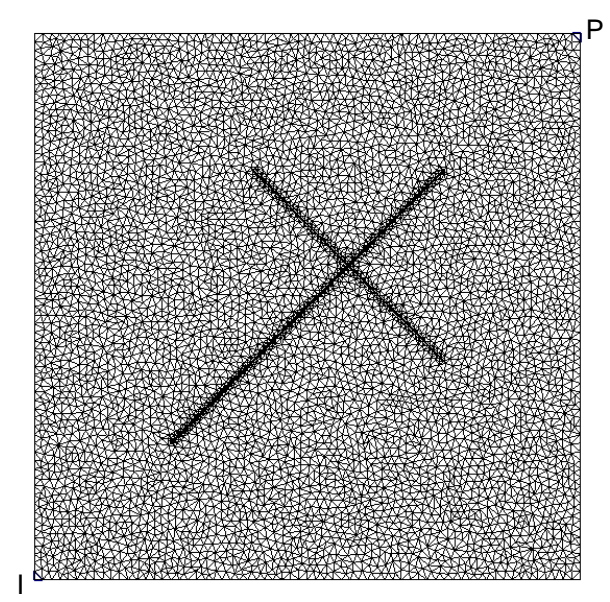

(a) 11984 cells

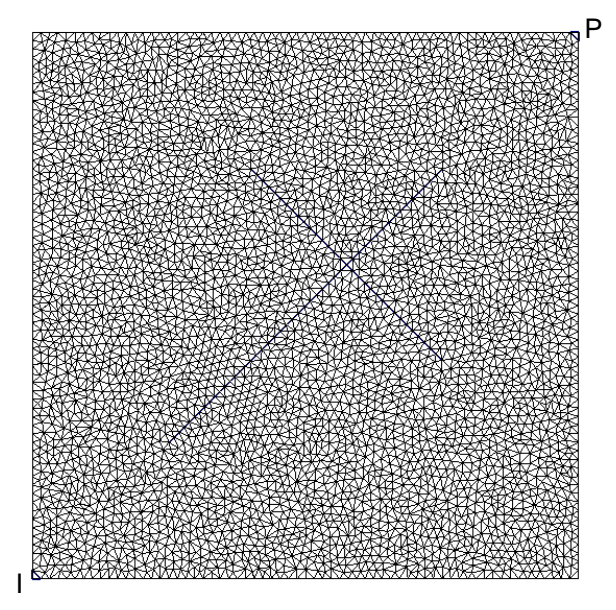

(b) 8648 cells $+801 \mathrm{D}$ fracture cells

Figure 20: a)Explicit grid representation of intersecting fractures of aperture $=10^{-3} \mathrm{~m}$ and b) mixed-dimensional grid with 1D fractures representation. Position of injector is marked by I and producer is marked by P.

\subsection{Numerical performance of the $1 D$ fracture model}

Tests to date indicate that approximately between $80-90 \%$ of the computational time is taken by the solution of the linear system. The linear systems are usually solved by the iterative solution methods. The performance of the iterative solution method is dependent on the properties of the system matrix $(G)$ to be solved and one of the important properties is the condition number $\left(\kappa=\| G|||| G^{-1}||\right)$. We present the comparison of condition number and the solution time of the global linear systems obtained by the 2D fracture model and the reduced-dimensional fracture model for a injector-producer problem solved over a domain with intersecting fractures and barriers(blocking fractures). The domain, of size $120 \mathrm{~m} \times 120 \mathrm{~m}$, is shown in the figure 25] The rock is assigned the identity permeability matrix, $\boldsymbol{K}_{m}=\boldsymbol{I} m D$. Fractures are assigned permeability of $\boldsymbol{K}_{\boldsymbol{f} \mathbf{1}}=k_{f} \boldsymbol{K}_{\boldsymbol{m}}$ while barriers are assigned permeability of $\boldsymbol{K}_{\boldsymbol{f} \mathbf{2}}=\left(1 / k_{f}\right) \boldsymbol{K}_{\boldsymbol{m}}$, where $k_{f}$ is the permeability contrast factor. We solve the problem for a range of aperture $(a)$ values $10^{-3} \mathrm{~m}$ and $10^{-2} m$ and fracture permeability values, $k_{f}=\left\{10^{4}, 10^{6}, 10^{7}\right\}$. In the $2 \mathrm{D}$ fracture model, fractures are gridded into $2 \mathrm{D}$ triangles as for the matrix. For the comparative study, we solve the problems on relatively coarser meshes which is for convenience of approximating condition numbers. The meshes are shown in figure 26. Note that, the fracture cell lengths along the fractures for both the meshes is equal unlike the previous section where fine Delaunay triangular cells of better quality are used in the 2D fractures which would increase the degrees of freedom in the fracture considerably. There are many more degrees of freedom for the 2D fractures, which also have cells of very small size at the intersection of the fractures, where as in the reduced-dimensional model the 1D fractures are modeled as triangle edges and the intersection cell is not included physically. Fluid is injected through the bottom-left cell at the rate of $1.5855 \mathrm{e} 2 \mathrm{~m}^{3} /$ year and the pressure of 100 bars is imposed at the top-right producer. We solve using the CVD-MPFA scheme with $q=2 / 3$, which gives an SPD system for the triangulated 2D fractures, whereas symmetry is lost locally 
2D fracture
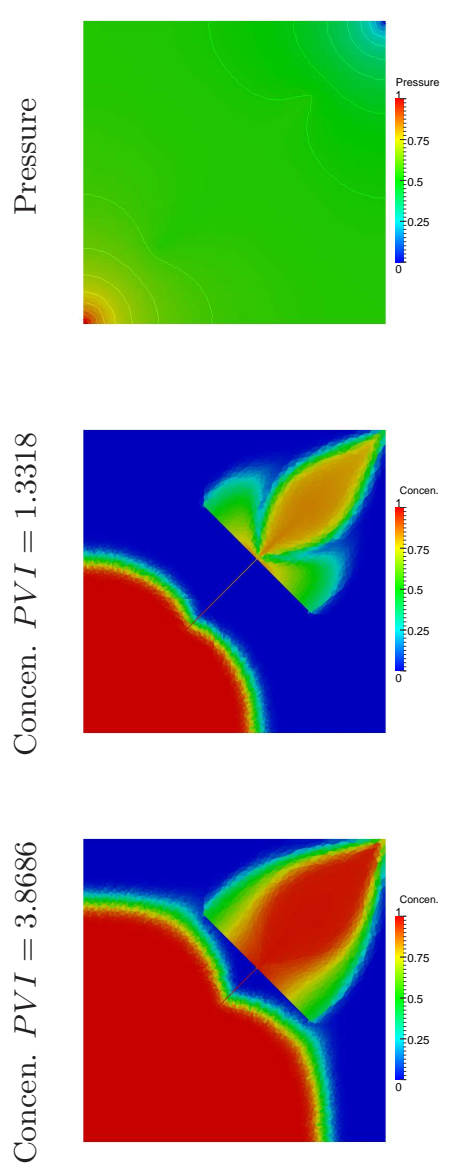

Hybrid-grid
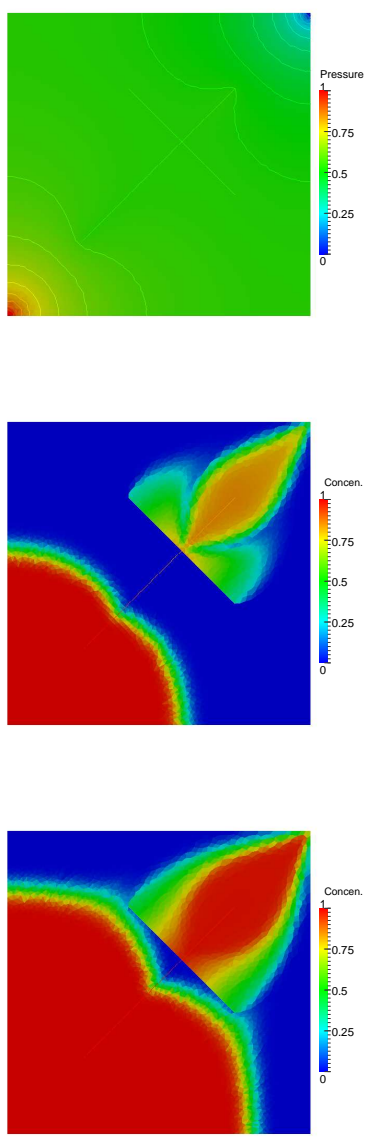

1D fracture
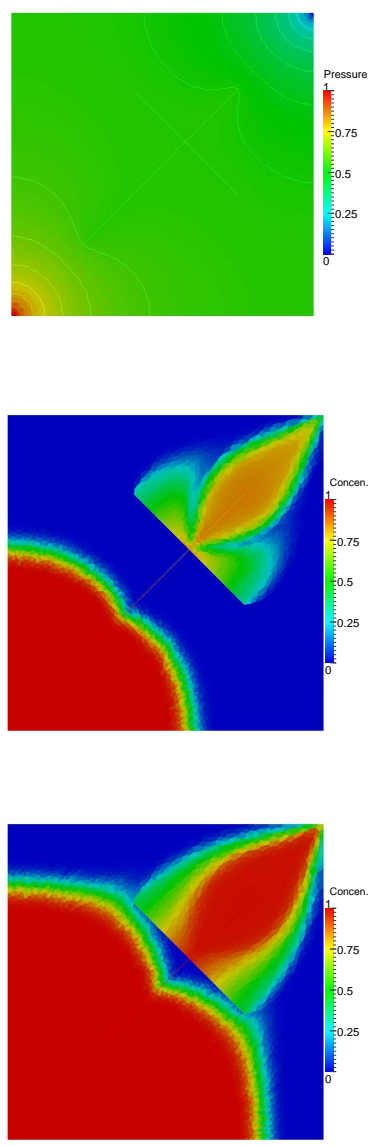

Figure 21: Pressure contours and tracer concentration contours at $P V I=1.3318$ and $P V I=3.8686$ by $2 \mathrm{D}$ fracture, hybrid-grid and $1 \mathrm{D}$ fracture model for isotropic permeability and $k_{f}=10^{4}$.

at a $1 \mathrm{D}$ fracture, because the quadrature $q=1$ is required in order to impose continuity in passing to the lower dimensional approximation, where a matrix cell has a fracture interface. We employ the library PETSc(v 3.5.2)[43] for the linear solution of the global systems and execute computations on the machine with Intel(R) Xeon(R) CPU E5-2687W v2 @3.40GHz. The global system for a 2D fracture is solved via the conjugate gradient $(\mathrm{CG})$ method, preconditioned by algebraic multi-grid(AMG) provided in PETSc. The system obtained by the 1D fracture model is solved via the generalized minimal residual(GMRES) method, preconditioned by AMG. Pressure solutions obtained by both the models are shown in figure27 for aperture, $a=10^{-3} \mathrm{~m}$ and $k_{f}=10^{6}$. Behavior of the solution of the 1D fracture model is in excellent agreement with the result of the $2 \mathrm{D}$ fracture model.

The condition number $(\kappa)$ of the global systems obtained by both the models and the respective CPU time for the linear solvers are shown in figures 28 and 29 for different values of $k_{f}$ and apertures. It is shown that the computational cost (condition number and the CPU time) for the 2D fracture model is more than for the 1D fracture model because of the higher number of global degrees of freedom, triangular cells of small 
2D fracture
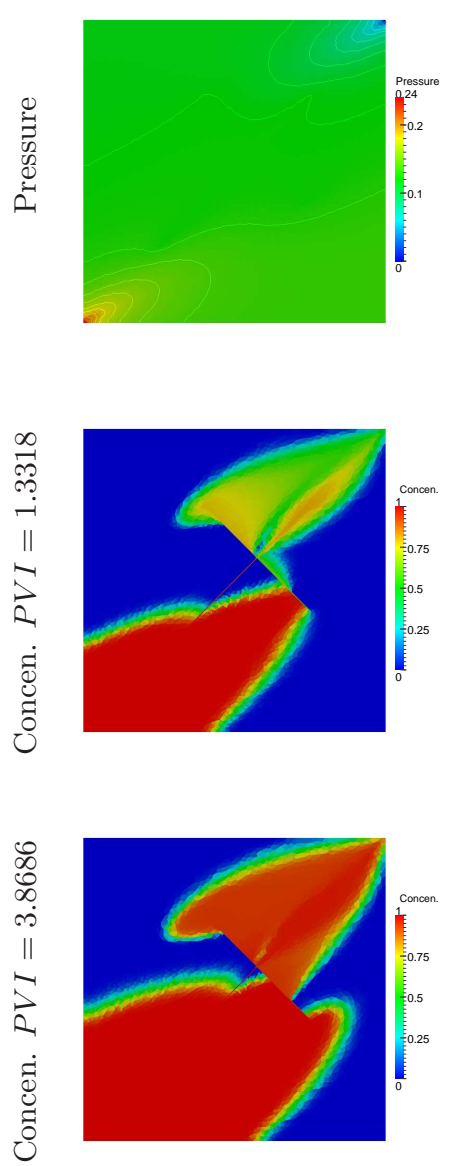

Hybrid-grid
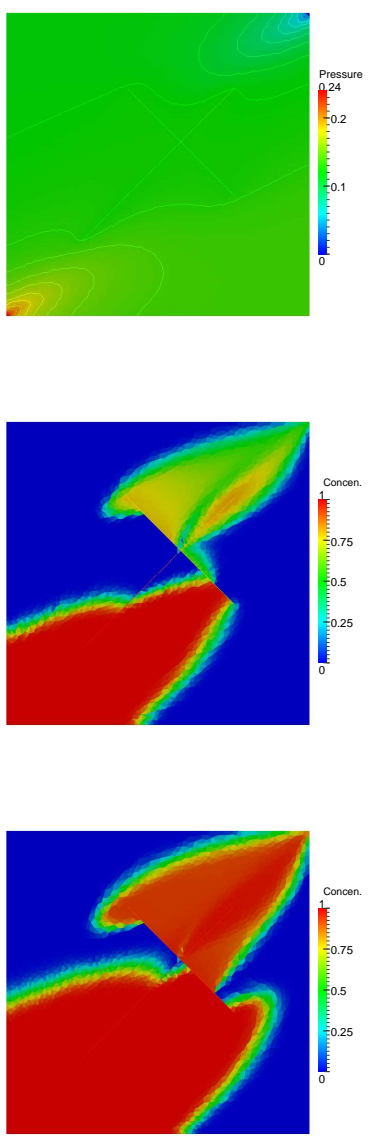

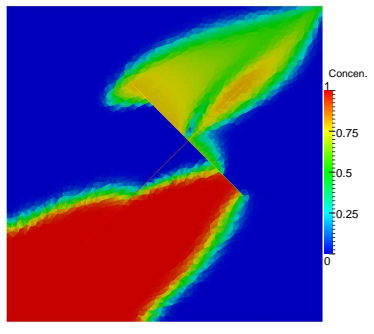

1D fracture
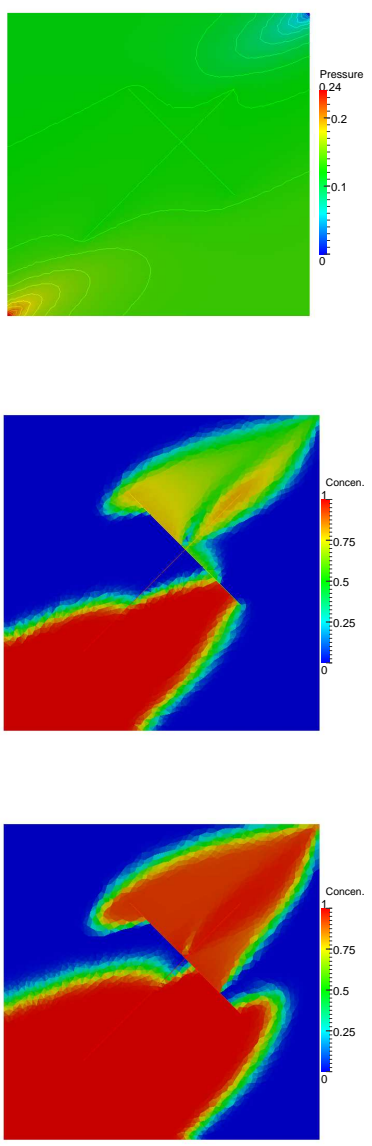

Figure 22: Pressure contours and tracer concentration contours at $P V I=1.3318$ and $P V I=3.8686$ by $2 \mathrm{D}$ fracture, hybrid-grid and 1D fracture model for anisotropic permeability and $k_{f}=10^{4}$.

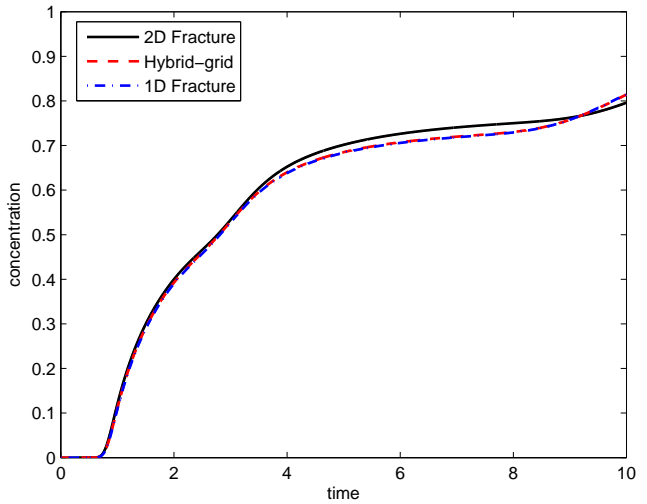

(a) Isotropic case

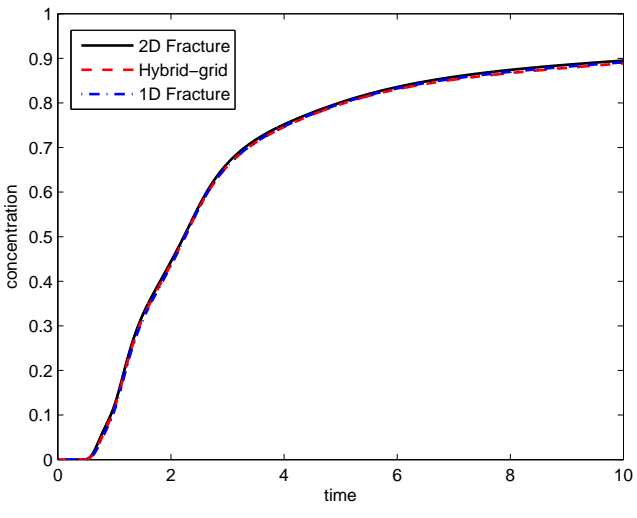

(b) Anisotropic case

Figure 23: Plots of tracer concentration at producer w.r.t time for permeability contrast $k_{f}=10^{4}$. 


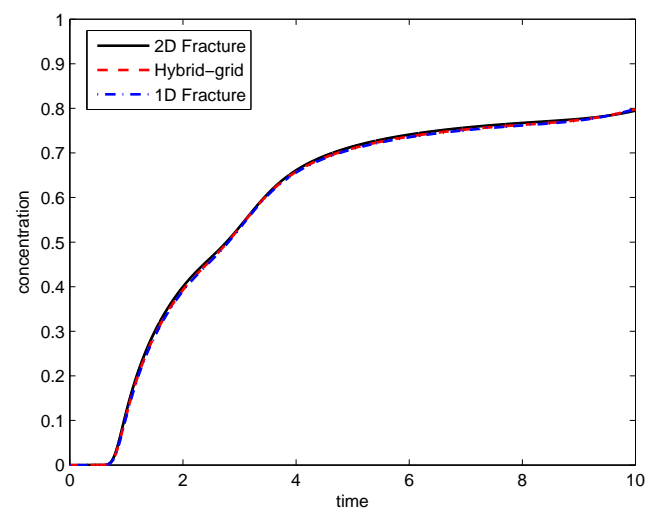

(a) Isotropic case

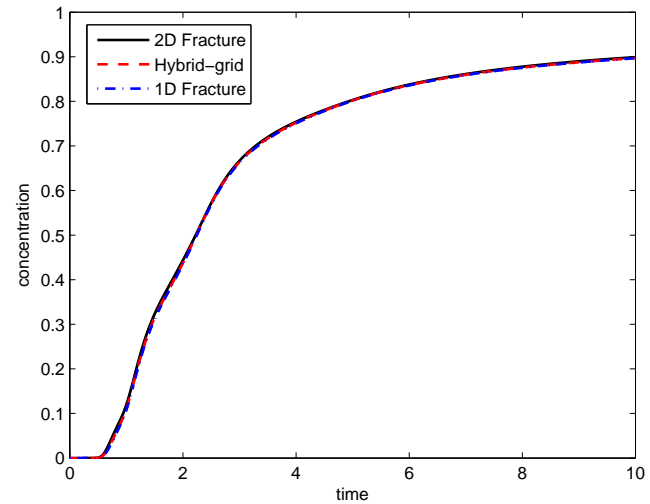

(b) Anisotropic case

Figure 24: Plots of tracer concentration at producer w.r.t time for permeability contrast $k_{f}=10^{6}$.

angles within 2D fractures and cells of small size(aperture) at junctions where 2D fractures intersect. The computational cost of the 2D fracture model increases with the increase of $k_{f}$ value much more than for the 1D fracture model. The AMG preconditioner performs very well for the solution of $1 \mathrm{D}$ fracture model with higher values of $k_{f}$ as well. The condition number of the 1D fracture for the aperture $a=10^{-3} \mathrm{~m}$ is less than for the $a=10^{-2} \mathrm{~m}$ and so is the computational cost. But, the computational cost for the 2D fractures is higher for the smaller value of aperture because of the small size of the intersection cell. These results show that global system with the 1D fracture model is better conditioned and relatively easier and quicker to solve without loosing considerable accuracy as compared to the 2D fracture. Note that, the order of magnitude of the condition number of the systems obtained by the hybrid-grid model is similar to the presented 1D fracture model. In our experience, if we use a quality refined Delaunay triangular mesh, so that there are regular triangles in the 2D fractures, the number of global degrees of freedom are very high and the linear system obtained for 2D fractures is so highly ill-conditioned that it is not of practical use. In addition, 1D fracture modeling simplifies the mesh generation involved and has less of a CFL restriction for the transport problems when solved explicitly in time. Moreover, the memory usage and computational cost for the local linear systems resulting from the cluster of cells (used in constructing the finite-volume CVD-MPFA fluxes) are also higher for the 2D fracture model than for the 1D fracture model.

\subsection{Transient pressure simulation with complex fracture network}

We solve a transient pressure equation for slightly compressible single phase-flow, governed by

$$
\varphi c_{t} \frac{\partial \phi}{\partial t}-\nabla \cdot \frac{\boldsymbol{k}}{\mu} \nabla \phi=q_{c}
$$




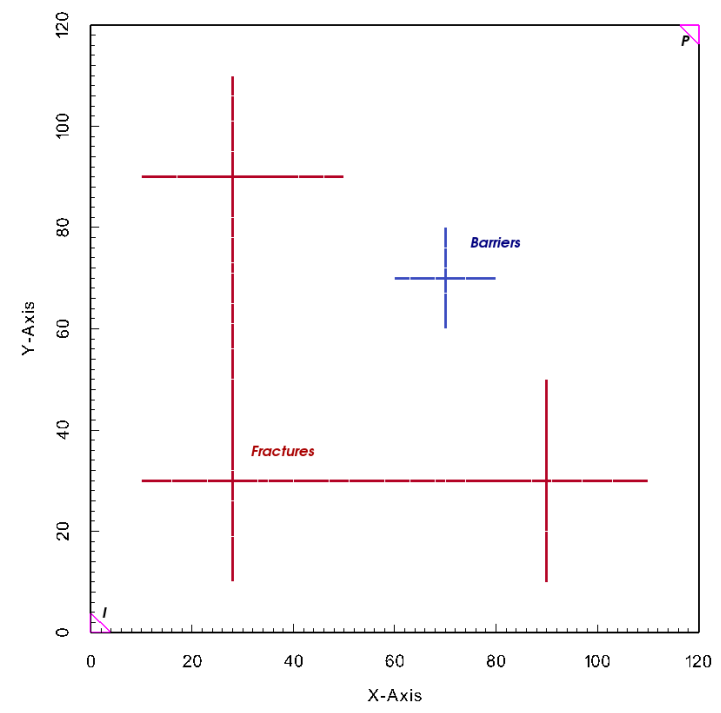

Figure 25: Domain with intersecting fractures and the barriers. The locations of injector and producer are also indicated.
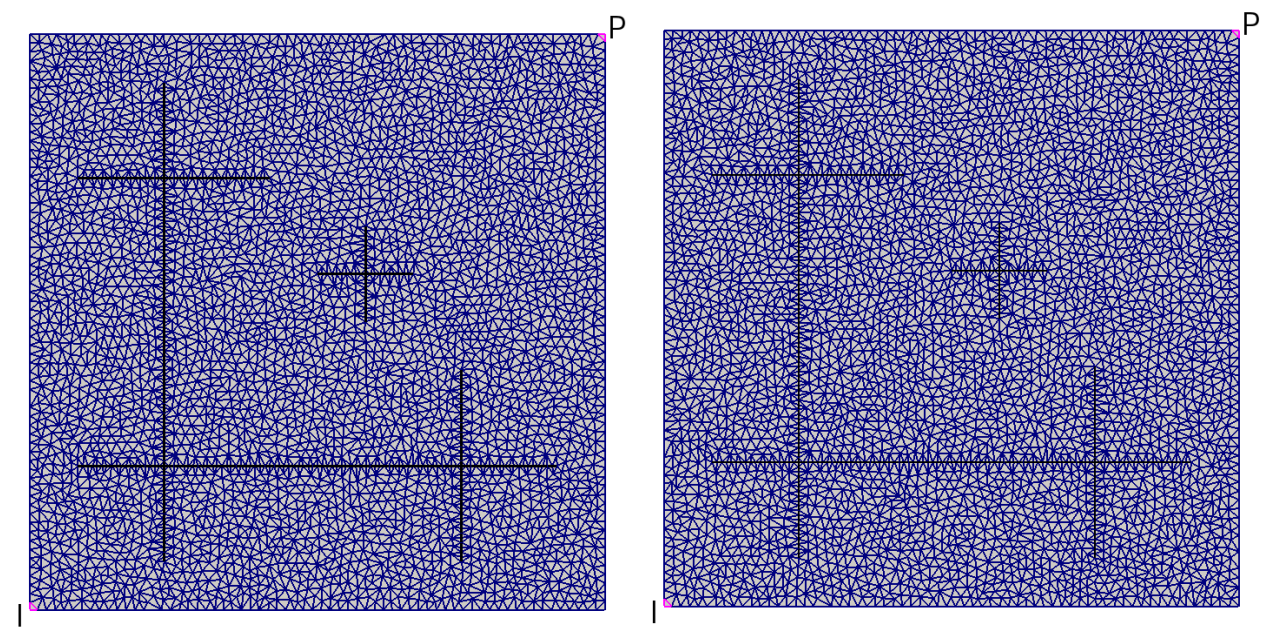

(a) Mesh with 2D gridded fractures. 8400 2D tri- (b) Mesh with 1D fractures. 7766 2D triangular angular cells cells $+3201 \mathrm{D}$ cells

Figure 26: a) Mesh with 2D gridded fractures and b) mixed-dimensional mesh with 1D fractures. Fracture cell length along the fracture in both meshes is equal.

where, $\varphi$ is the porosity of domain and $c_{t}$ is the total compressibility which is assumed to be constant. The discrete form of the equation(42), using (39), for the implicit scheme (in time), can be written as;

$$
\left(\begin{array}{cc}
M_{m}+G_{m m} & \mathcal{G}_{m f} \\
\mathcal{G}_{T m} & M_{f}+\bar{G}_{f f}
\end{array}\right)\left(\begin{array}{c}
\Phi_{m}^{n+1} \\
\Phi_{f}^{n+1}
\end{array}\right)=\left(\begin{array}{c}
\overline{\boldsymbol{q}}_{c m}+M_{m} \Phi_{m}^{n} \\
\overline{\boldsymbol{q}}_{c f}+M_{f} \Phi_{f}^{n}
\end{array}\right) \quad \text { for time step } n
$$

where, $M_{m}$ and $M_{f}$ are diagonal systems of coefficients $\varphi_{m} c_{t} / \delta t, \varphi_{f} c_{t} / \delta t$ associated with matrix and fracture respectively. The domain size is $500 \mathrm{~m} \times 420 \mathrm{~m}$ which includes a complex fracture network consisting of 1055 interconnected fracture segments. A producer is located in the middle of the domain containing the fracture 


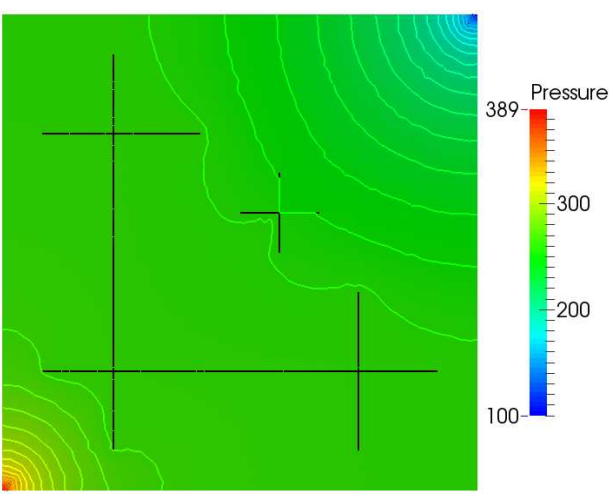

(a) Pressure solution for 2D gridded fractures

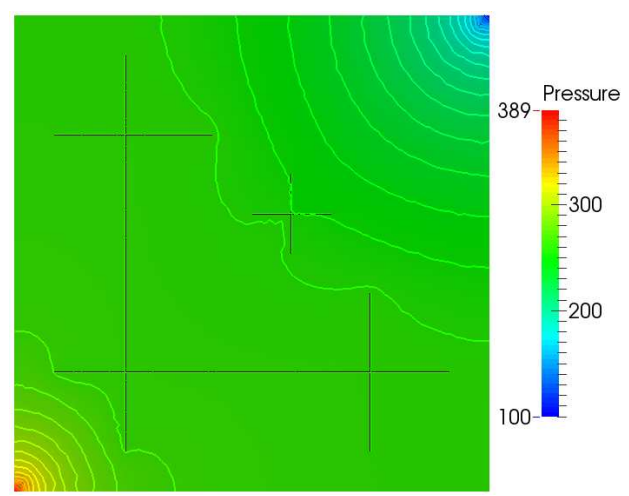

(b) Pressure solution for 1D fractures

Figure 27: Pressure solutions given by 2D fracture and 1D fracture models for aperture, $a=10^{-3} \mathrm{~m}$ and $k_{f}=10^{6}$.

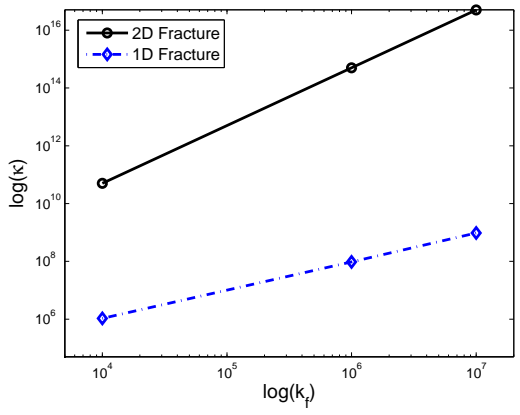

(a) Condition number $\kappa$

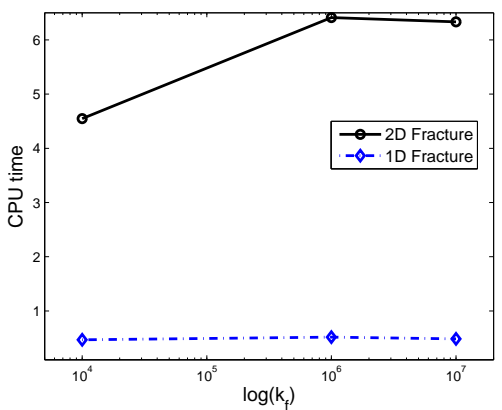

(b) CPU time

Figure 28: Condition number $(\kappa)$ and CPU time in sec for the 2D fracture and 1D fracture models for aperture, $a=10^{-3} \mathrm{~m}$.

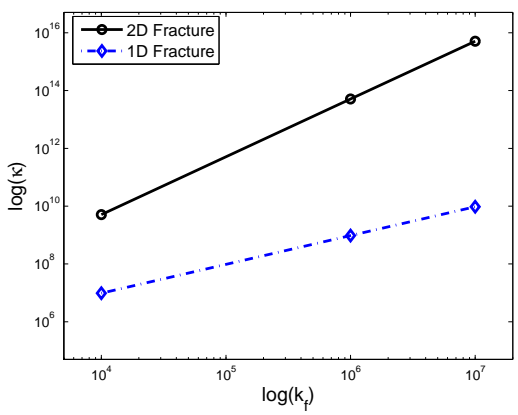

(a) Condition number $\kappa$

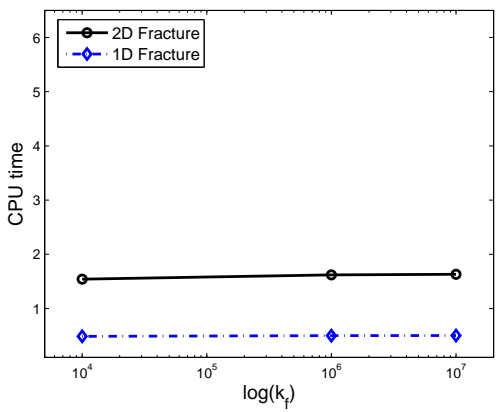

(b) CPU time

Figure 29: Condition number $(\kappa)$ and CPU time in sec for the 2D fracture and 1D fracture models for aperture, $a=10^{-2} m$.

network, as shown in figure30. The producer is intersecting sections of the fracture network and the matrix. The producer is set at constant pressure of 100bars. The initial pressure of the whole reservoir is 300bars. We discretize the whole domain into a conforming unstructured triangular mesh with interior triangle edges aligned with the fractures. A fracture conforming 2D mesh is generated using Triangle [41]. The mesh is shown in figur 31 Due to the complexity and high density of the fracture network, the grid contains some 
Figure 30: Highly fractured domain. The complex fracture network include 1055 interconnected fracture segments. A producer $(\mathrm{P})$ is also depicted in the center of the domain.

\section{Conclusions}

We have presented a CVD-MPFA formulation for discrete fracture-matrix simulations where lowerdimensional fracture networks are efficiently coupled with CVD-MPFA for the rock matrix. We have compared pressure and transport results obtained by the lower-dimensional fracture model, hybrid-grid method and equi-dimensional model on unstructured meshes. For thin highly conductive fractures, the lower-dimensional fracture model with continuous pressure approximation across the fracture yields results 


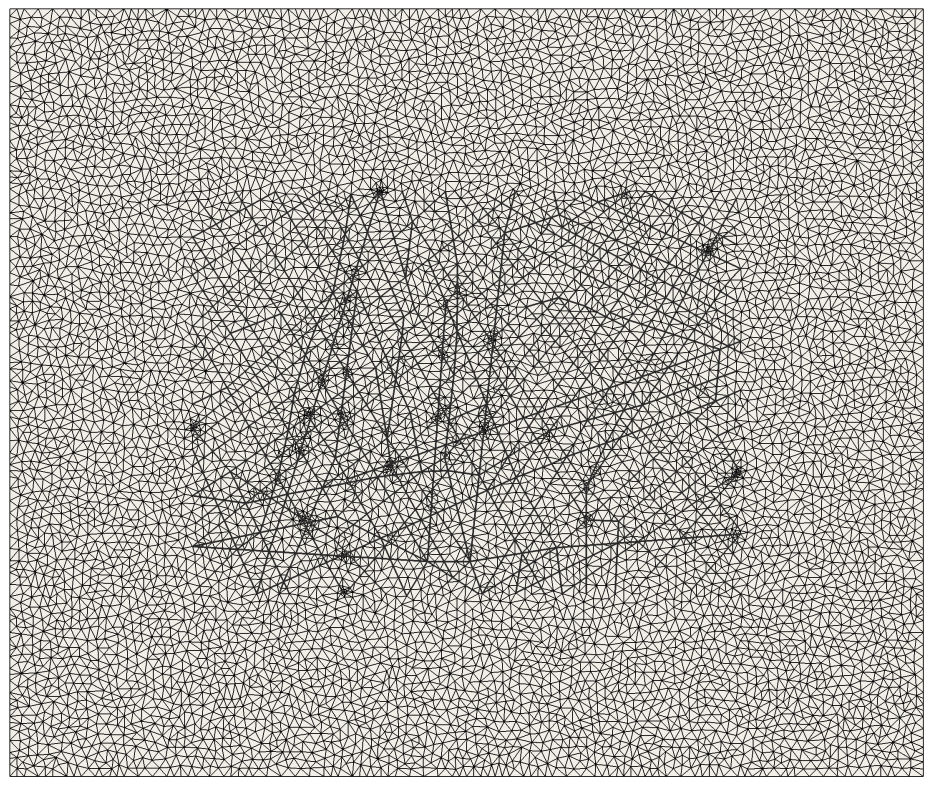

Figure 31: Mixed dimensional mesh of the fractured domain. 18295 triangles +3082 lines

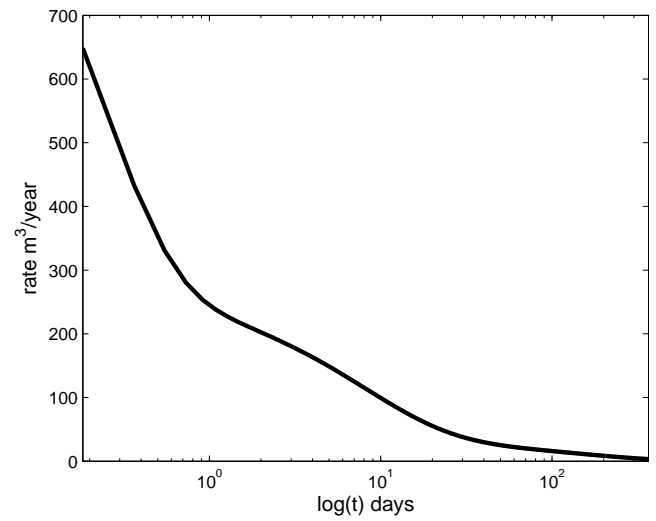

Figure 32: Producer rate for the transient pressure solution over the domain involving discrete fracture-matrix system for a period of 1year.

that rival those of the hybrid-grid method and explicit equi-dimensional modeling of fractures, without including extra matrix-fracture interfaces, thus reducing the local degrees of freedom. Numerical tests show that the lower-dimensional model with discontinuous pressure across the fracture yields improved flow resolution when compared to the hybrid-grid method for low permeability in the direction normal to the fracture, while maintaining a simpler equation set at the fracture. We also note that in the limit, for high normal permeability, the transmission conditions return to continuity of pressure.

A transient pressure simulation is also presented for a more complex discrete fracture-matrix system, which demonstrates the applicability of the method for the multi-rate aspects of drainage of a fractured zone.

For problems involving systems of thin highly conductive fractures, we recommend the lower-dimensional fracture model with continuous pressure approximation. Problems involving barriers are modeled by the 


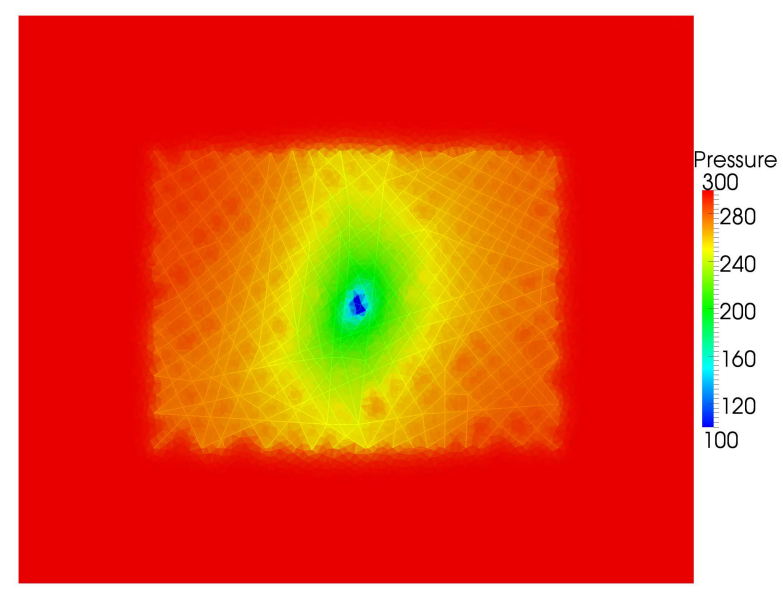

(a) 02 days

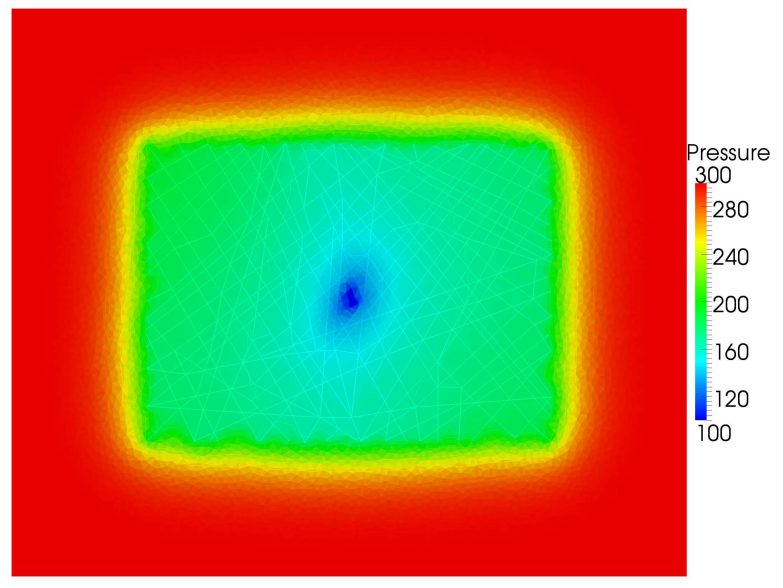

(b) 13 days

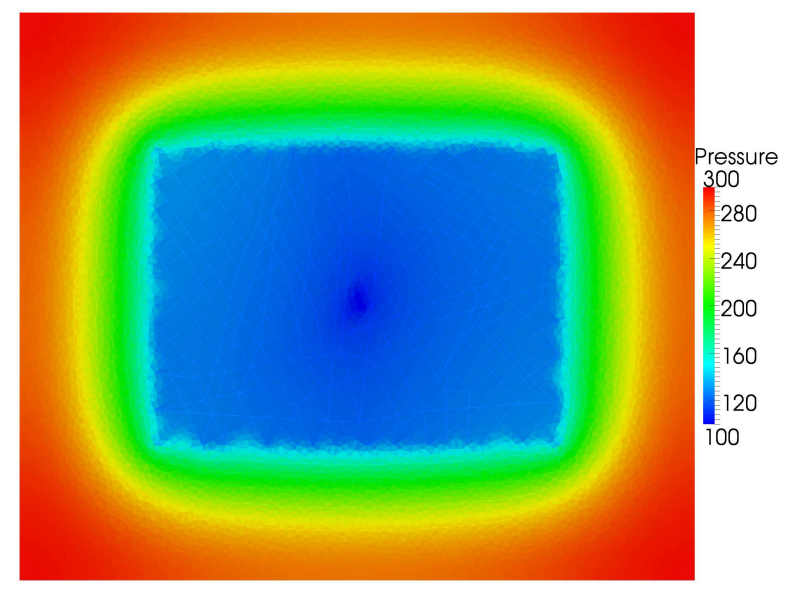

(c) 46 days

Figure 33: Pressure contours at three different times of the transient pressure solution.

lower-dimensional model with discontinuous pressure approximation and setting the parameter $\zeta=2.0 / 3.0$.

The new formulation can easily be implemented within any existing CVD-MPFA simulator coupled with a DFN solver.

\section{Acknowledgements}

We thank Shell Global Solutions International B.V., Rijswijk, the Netherlands, for the financial support for this work. We also thank the anonymous reviewers for very constructive comments.

\section{References}

[1] S. K. Matthäi, Modelling multiphase flow in fractured porous rock, Tech. rep., Department of Earth Sciences \& Engineering, Imperial College London (2005).

[2] H. Hoteit, A. Firoozabadi, An efficient numerical model for incompressible two-phase flow in fractured media, Adv. Water Resour. 31 (06) (2008) 891-905. 
[3] L. H. Reiss, The reservoir engineering aspects of fractured formations, Editions Technip, Paris, 1980.

[4] J. Erhel, J.-R. D. Dreuzy, B. Poirriez, Flow simulation in three-dimensional discrete fracture networks., SIAM J. Sci. Comput. 31 (04) (2009) 2688-2705.

[5] B. Berkowitz, Characterizing flow and transport in fractured geological media: A review, Adv. Water Resour. 25 (2002) 861-884.

[6] J. E. P. Monteagudo, A. Firoozabadi, Control-volume method for numerical simulation of two-phase immiscible flow in two- and three-dimensional discrete-fractured media, Water Resour. Res. 40 (07).

[7] G. Barenblatt, Y. Zheltov, I. Kochina, Basic concepts in the theory of seepage of homogeneous fluids in fissurized rocks, PMM 24 (05) (1960) 852-864.

[8] J. Warren, P. Root, The behavior of naturally fractured reservoirs, SPE Journal 03 (03) (1963) $245-255$.

[9] H. Kazemi, L. S. Merrill, K. L. Porterfield, P. R. Zeman, Numerical simultion of water-oil flow in naturally fractured reservoirs, SPE Journal 16 (06) (1976) 317-326.

[10] Z. Chen, G. Huan, Y. Ma, Computational Methods for Multi-phase Flow in Porous Media, Society for Industrial and Applied Mathematics, 2006.

[11] J. Noorishad, M. Mehran, An upstream finite element method for solution of transient transport equation in fractured porous media, Water Resour. Res. 18 (03) (1982) 588-596.

[12] R. Baca, R. Arnett, D. Langford, Modeling fluid flow in fractured porous rock masses by finite element techniques, Int. J. Numer. Methods Fluids 04 (04) (1984) 337-348.

[13] M. F. Lough, S. H. Lee, J. Kamath, An efficient boundary integral formulation for flow through fractured porous media, J. Comput. Phys. 143 (2) (1998) 462-483.

[14] M. Karimi-Fard, L. J. Durlofsky, K. Aziz, An efficient discrete-fracture model applicable for general-purpose reservoir simulators, SPE Journal 09 (02) (2004) 227-236.

[15] S. K. Matthäi, A. Mezentsev, M. Belayneh, Control-volume finite-element two-phase flow experiments with fractured rock represented by unstructured 3d hybrid meshes, in: SPE Reservoir Simulation Symposium, Society of Petroleum Engineers, 2005.

[16] V. Martin, J. Jaffré, J. E. Roberts, Modelling fractures and barriers as interfaces for flow in porous media, SIAM J. Sci. Comput. 26 (2005) 1667-1691.

[17] H. Hægland, A. Assteerawatt, H. K. Dahle, G. T. Eigestad, R. Helmig, Comparison of cell- and vertex-centered discretization methods for flow in a two-dimensional discrete-fracture-matrix system, Adv. Water Resour. 32 (12) (2009) 1740-1755.

[18] T. H. Sandve, I. Berre, J. M. Nordbotten, An efficient multi-point flux approximation method for discrete fracture-matrix simulations, J. Comput. Phys. 231 (09) (2012) 3784-3800.

[19] B. T. Mallison, M. H. Hui, W. Narr, Practical gridding algorithms for discrete fracture modeling workflows, in: 12th European Conference on the Mathematics of Oil Recovery, EAGE, 2010.

[20] H. Mustapha, R. Dimitrakopoulos, T. Graf, A. Firoozabadi, An efficient method for discretizing 3D fractured media for subsurface flow and transport simulations, Int. J. Numer. Methods Fluids 67 (5) (2011) 651-670.

[21] H. Hajibeygi, Iterative multiscale finite volume method for multiphase flow in porous media with complex physics, Ph.D. thesis, ETH Zurich (2011).

[22] E. Unsal, S. K. Matthäi, M. J. Blunt, Simulation of multiphase flow in fractured reservoirs using a fracture-only model with transfer functions, Computat. Geosci. 14 (2010) 527-538.

[23] S. H. Lee, M. F. Lough, C. L. Jensen, Hierarchical modeling of flow in naturally fractured formations with multiple length scales, Water Resour. Res. 37 (03) (2001) 443-455.

[24] H. Hajibeygi, D. Karvounis, P. Jenny, A hierarchical fracture model for the iterative multiscale finite-volume method, J. Comput. Phys. 230 (24) (2011) 8729-8743. 
[25] C. D. Foster, T. M. Nejad, Embedded discontinuity finite element modeling of fluid flow in fractured porous media, Acta Geotechnica 8 (1) (2013) 49-57.

[26] C. D'Angelo, A. Scotti, A mixed finite element method for Darcy flow in fractured porous media with nonmatching grids, ESAIM: Math. Model. Numer. Anal. 46 (2012) 465-489.

[27] S. Berrone, S. Pieraccini, S. Scialò, An optimization approach for large scale simulations of discrete fracture network flows, J. Comput. Phys. 256 (2014) 838-853.

[28] A. Fourno, C. Grenier, A. Benabderrahmane, F. Delay, A continuum voxel approach to model flow in 3D fault networks: A new way to obtain up-scaled hydraulic conductivity tensors of grid cells, J. Hydrol. 493 (2013) 68-80.

[29] B. Noetinger, N. Jarrige, A quasi steady state method for solving transient Darcy flow in complex 3D fractured networks, J. Comput. Phys. 231 (1) (2012) 23-38.

[30] H. A. Friis, M. G. Edwards, J. Mykkeltveit, Symmetric positive definite flux-continuous full-tensor finite-volume schemes on unstructured cell-centered triangular grids, SIAM J. Sci. Comput. 31 (02) (2008) 1192-1220.

[31] M. G. Edwards, C. F. Rogers, Finite volume discretization with imposed flux continuity for the general tensor pressure equation, Computat. Geosci. 02 (04) (1998) 259-290.

[32] M. Pal, M. G. Edwards, q-families of CVD(MPFA) schemes on general elements: Numerical convergence and the maximum principle, Arch. Comput. Methods Eng. 17 (2010) 137-189.

[33] I. Aavatsmark, An introduction to multipoint flux approximations for quadrilateral grids, Computat. Geosci. $06(3-4)(2002)$ 405-432.

[34] M. G. Edwards, H. Zheng, A quasi-positive family of continuous Darcy-flux finite-volume schemes with full pressure support, J. Comput. Phys. 227 (22) (2008) 9333-9364.

[35] M. G. Edwards, H. Zheng, Double-families of quasi-positive Darcy-flux approximations with highly anisotropic tensors on structured and unstructured grids, J. Comput. Phys. 229 (03) (2010) 594-625.

[36] H. A. Friis, M. G. Edwards, A family of MPFA finite-volume schemes with full pressure support for the general tensor pressure equation on cell-centered triangular grids, J. Comput. Phys. 230 (01) (2011) 205-231.

[37] M. Karimi-Fard, A. Firoozabadi, Numerical simulation of water injection in 2D fractured media using discretefracture model, in: SPE Annual Technical Conference and Exhibition, 2001.

[38] P. Angot, F. Boyer, F. Hubert, Asymptotic and numerical modelling of flows in fractured porous media, ESAIM: Math. Model. Numer. Anal. 43 (02) (2009) 239-275.

[39] F. Heße, V. Prykhodko, S. Attinger, Assessing the validity of a lower-dimensional representation of fractures for numerical and analytical investigations, Adv. Water Resour. 56 (2013) 35-48.

[40] V. Reichenberger, H. Jakobs, P. Bastian, R. Helmig, A mixed-dimensional finite volume method for two-phase flow in fractured porous media, Adv. Water Resour. 29 (07) (2006) 1020-1036.

[41] J. R. Shewchuk, Triangle: Engineering a 2D Quality Mesh Generator and Delaunay Triangulator, in: Applied Computational Geometry: Towards Geometric Engineering, Vol. 1148 of Lecture Notes in Computer Science, Springer-Verlag, 1996, pp. 203-222.

[42] J.-G. Kim, M. D. Deo, Finite element, discrete-fracture model for multiphase flow in porous media, AIChE Journal 46 (06) (2000) 1120-1130.

[43] S. Balay, M. F. Adams, J. Brown, P. Brune, K. Buschelman, V. Eijkhout, W. D. Gropp, D. Kaushik, M. G. Knepley, L. C. McInnes, K. Rupp, B. F. Smith, H. Zhang, PETSc Web page, http://www.mcs.anl.gov/petsc (2014). 